\title{
A NEW THEORY OF FRACTIONAL DIFFERENTIAL CALCULUS*
}

\author{
XIAOBING FENG $^{\dagger}$ AND MITCHELL SUTTON $\ddagger$
}

\begin{abstract}
This paper presents a self-contained new theory of weak fractional differential calculus in one-dimension. The crux of this new theory is the introduction of a weak fractional derivative notion which is a natural generalization of integer order weak derivatives; it also helps to unify multiple existing fractional derivative definitions and characterize what functions are fractionally differentiable. Various calculus rules including a fundamental theorem calculus, product and chain rules, and integration by parts formulas are established for weak fractional derivatives. Additionally, relationships with classical fractional derivatives and detailed characterizations of weakly fractional differentiable functions are also established. Furthermore, the notion of weak fractional derivatives is also systematically extended to general distributions instead of only to some special distributions. This new theory lays down a solid theoretical foundation for systematically and rigorously developing new theories of fractional Sobolev spaces, fractional calculus of variations, and fractional PDEs as well as their numerical solutions in subsequent works. This paper is a concise presentation of the materials of Sections 1-4 and 6 of reference [9].
\end{abstract}

Key words. Weak fractional derivatives, fractional differential calculus, fundamental theorem of calculus, product and chain rules, fractional derivatives of distributions.

AMS subject classifications. 26A33, 34K37, 35R11, 46E35,

1. Introduction. Similar to the classical integer order calculus, the classical fractional order calculus also consists of two integral parts, namely, the fractional order integral calculus and the fractional order differential calculus. It is concerned with studying their properties/rules and the interplay between the two notions, which is often characterized by the so-called Fundamental Theorem of Calculus. Fractional calculus also has had a long history, which can be traced back to L'Hôpital (1695), Wallis (1697), Euler (1738), Laplace (1812), Lacroix (1820), Fourier (1822), Abel (1823), Liouville (1832), Riemann (1847), Leibniz (1853), Grünwald (1867), Letnikov (1868) and many others. We refer the reader to [14, 28, 30] and the references therein for a detailed exposition about the history of the classical fractional calculus.

In the past twenty years fractional calculus and fractional (order) differential equations have garnered a lot of interest and attention both from the PDE community (in the name of nonlocal PDEs) and in the applied mathematics and scientific communities. Besides the genuine mathematical interest and curiosity, this trend has also been driven by intriguing scientific and engineering applications which give rise to fractional order differential equation models to better describe the (time) memory effect and the (space) nonlocal phenomena (cf. 66, 14, 16, 18, 23, and the references therein). It is the rise of these applications that revitalizes the field of fractional calculus and fractional differential equations and calls for further research in the field, including to develop new numerical methods for solving various fractional order problems.

Although a lot of progress has been achieved in the past twenty years in the field of fractional calculus and fractional differential equations, many fundamental issues remain to be addressed. For a novice in the field, one would immediately be clogged and confused by many (non-equivalent) definitions of fractional derivatives. The immediate ramification of the situation is the difficulty for building/choosing "correct"

\footnotetext{
${ }^{*}$ This work was partially supported by the NSF grant 1620168 .

$\dagger$ Department of Mathematics, The University of Tennessee, Knoxville, TN 37996. U.S.A. (xfeng@math.utk.edu).

${ }^{\ddagger}$ Department of Mathematics, The University of Tennessee, Knoxville, TN 37996. U.S.A. (msutto11@vols.utk.edu).
} 
fractional models to study analytically and to solve numerically. Moreover, compared to the classical integer order calculus, the classical fractional calculus still has many missing components. For example, many basic calculus rules (such as product and chain rules) are not completely settled, the physical and geometric meaning of fractional derivatives are not fully understood, and a thorough characterization of the fractional differentiability seems still missing. Furthermore, at the differential equation (DE) level, the gap between the integer order and fractional order cases is even wider. For a given integer order DE, it would be accustomed for one to interpret the derivatives in the DE as weak derivatives and the solution as a weak solution. However, there is no parallel weak derivative and solution theory in the fractional order case so far. As a result, various solution concepts and theories, which may not be equivalent, have been used for fractional order DEs, especially, for fractional (order) partial differential equations (FPDEs). The non-equivalence of solution concepts may cause confusions and misunderstanding of the underlying fractional order problem.

The primary goal of this paper is to develop a new weak fractional differential calculus theory, starting from introducing the definition, proving various calculus rules, to establishing the Fundamental Theorem of Weak Fractional Calculus (FTwFC). These results lay down the ground work for developing a new fractional Sobolev space theory in a companion paper [10]. Together they provide a first step/attempt in achieving the overreaching goal of providing the missing components to, and to expand the reach of, the classical fractional calculus and fractional differential equations, which will be continued in subsequent works [11, 12.

The remainder of this paper is organized as follows. In Section 2, we recall the definitions of some well-known classical fractional derivatives and a few relevant properties, which all can be found in the beginning chapters of the reference [30] (also see [9, Section 2]). Additionally, we present an alternative perspective of the classical fractional derivatives. This is to introduce the so-called Fundamental Theorem of Classical Fractional Calculus (FTcFC) and a new interpretation/definition of the classical Riemann-Liouville fractional derivatives. Such a viewpoint is essential to developing the weak fractional calculus theory in this paper and the fractional Sobolev space theory in 10. In Section 3, we first introduce the notion of weak fractional derivatives using integration by parts and special test functions, which is analogous to the notion of integer order weak derivatives. It is proved that weak fractional derivatives inherit the fundamental properties of classical fractional derivatives and the generality eliminates the need for numerous definitions as seen in the classical theory. After having proved a characterization result, we then establish the FTwFC, product and chain rules, and integration by parts formulas. Many of these results and their proof techniques will look familiar to the informed reader because they are adapted and refined from those used in the integer order weak differential calculus theory (cf. [1, 8, 25]). The desired differential calculus components are proved for both left and right weak fractional derivatives and covers both finite and infinite domain cases. In Section 4, we extend the notion of weak fractional derivatives to distributions. Unlike the existing fractional derivative definitions which only apply to a certain subset of distributions, we aim to define weak fractional derivatives for general distributions. Due to the pollution effect of the fractional derivatives, the main difficulty to overcome is to design a good domain extension for a given distribution, which is achieved by using a partition of the unity idea in this section. Finally, the paper is concluded by a short summary and a few concluding remarks given in Section 5 . 
2. Preliminaries. In this section we first recall definitions of classical fractional integrals and derivatives without stating their well-known properties, we refer the interested reader to [9] for a collection of useful properties, and to 30] for a more extensive collection and their detailed proofs. We then present a couple lesser known properties of classical fractional derivatives which will be crucially and repeatedly used in the subsequent sections. One of which is the pollution behavior of classical fractional derivatives of compactly supported smooth function, the other is an equivalent definition of the Riemann-Liouville fractional derivatives based on the FTcFC.

Since the definitions of fractional integrals and derivatives are domain-dependent, it will be imperative for us to separate the cases when the domain is finite or infinite. In this section and in the later sections, we shall consider both finite interval $\Omega=(a, b)$ for $\infty<a<b<\infty$ and the infinite interval $\Omega=\mathbb{R}:=(-\infty, \infty)$.

Throughout this paper, $\Gamma: \mathbb{R} \rightarrow \mathbb{R}$ denotes the standard Gamma function and $\mathbb{N}$ stands for the set of all positive integers. In addition, $C$ will be used to denote a generic positive constant which may be different at different locations and $f^{(n)}$ denotes the $n$th order classical derivative of $f$ for $n \in \mathbb{N}$. Unless stated otherwise, all integrals $\int_{a}^{b} \varphi(x) d x$ are understood as Riemann integrals in this section.

2.1. Definitions of Classical Fractional Integrals and Derivatives. In this subsection, we recall the definitions of classical Riemann-Liouville, Caputo, GrünwaldLetnikov, and Fourier fractional integrals and derivatives.

2.1.1. Definitions on a Finite Interval. Historically, the integral calculus was invented before the differential calculus in the classical Newton-Leibniz (integer) calculus and the two are intimately connected through the well known Fundamental Theorem of Calculus (or Newton-Leibniz Theorem). It is interesting to note that the same is true for the classical fractional calculus. Indeed, in order to give a definition of fractional derivatives, we first need to recall the definition of fractional integrals.

Definition 2.1 (cf. 30]). Let $\sigma>0$ and $f:[a, b] \rightarrow \mathbb{R}$. The $\sigma$ order left Riemann-Liouville fractional integral of $f$ is defined by

$$
{ }_{a} I_{x}^{\sigma} f(x):=\frac{1}{\Gamma(\sigma)} \int_{a}^{x} \frac{f(y)}{(x-y)^{1-\sigma}} d y \quad \forall x \in[a, b],
$$

and the $\sigma$ order right Riemann-Liouville fractional integral of $f$ is defined by

$$
{ }_{x} I_{b}^{\sigma} f(x):=\frac{1}{\Gamma(\sigma)} \int_{x}^{b} \frac{f(y)}{(y-x)^{1-\sigma}} d y \quad \forall x \in[a, b] .
$$

${ }_{a} I_{x}^{\sigma}$ and ${ }_{x} I_{b}^{\sigma}$ are respectively called the left and right Riemann-Liouville fractional integral operators. We also set ${ }^{-} I^{\sigma}:={ }_{a} I_{x}^{\sigma}$ and ${ }^{+} I^{\sigma}:={ }_{x} I_{b}^{\sigma}$.

REMARK 2.1. It is well known ([30]) that both ${ }_{a} I_{x}^{\sigma}$ and ${ }_{x} I_{b}^{\sigma}$ are convolution-type operators (with different kernel functions).

With the help of the above fractional integrals, the definitions of two popular Riemann-Liouville fractional derivatives are given below.

Definition 2.2 (cf. [30]). Let $n-1<\alpha<n$ and $f:[a, b] \rightarrow \mathbb{R}$. The $\alpha$ order left Riemann-Liouville fractional derivative of $f$ is defined by

$$
\begin{aligned}
{ }_{a} D_{x}^{\alpha} f(x): & =\frac{d^{n}}{d x^{n}}\left({ }_{a} I_{x}^{n-\alpha} f(x)\right) \\
& =\frac{1}{\Gamma(n-\alpha)} \frac{d^{n}}{d x^{n}} \int_{a}^{x} \frac{f(y)}{(x-y)^{1+\alpha-n}} d y \quad \forall x \in[a, b],
\end{aligned}
$$


and the $\alpha$ order right Riemann-Liouville fractional derivative of $f$ is defined by

$$
\begin{aligned}
{ }_{x} D_{b}^{\alpha} f(x): & =(-1)^{n} \frac{d^{n}}{d x^{n}}\left({ }_{x} I_{b}^{n-\alpha} f(x)\right) \\
& =\frac{(-1)^{n}}{\Gamma(n-\alpha)} \frac{d^{n}}{d x^{n}} \int_{x}^{b} \frac{f(y)}{(y-x)^{1+\alpha-n}} d y \quad \forall x \in[a, b] .
\end{aligned}
$$

${ }_{a} D_{x}^{\alpha}$ and ${ }_{x} D_{b}^{\alpha}$ are called the left and right Riemann-Liouville fractional derivative(or differential) operators, respectively.

Another fractional derivative notion is the Caputo fractional derivative, which is widely used in initial value problems of factional order ODEs; particularly for fractional differentiation in time.

Definition 2.3 (cf. [30]). Let $n-1<\alpha<n$ and $f:[a, b] \rightarrow \mathbb{R}$. The $\alpha$ order left Caputo fractional derivative of $f$ is defined by

$$
{ }_{a}^{C} D_{x}^{\alpha} f(x):=\frac{1}{\Gamma(n-\alpha)} \int_{a}^{x} \frac{f^{(n)}(y)}{(x-y)^{1+\alpha-n}} d y \quad \forall x \in[a, b],
$$

and the $\alpha$ order right Caputo fractional derivative of $f$ is defined by

$$
{ }_{x}^{C} D_{b}^{\alpha} f(x):=\frac{(-1)^{n}}{\Gamma(n-\alpha)} \int_{x}^{b} \frac{f^{(n)}(y)}{(y-x)^{1+\alpha-n}} d y \quad \forall x \in[a, b]
$$

REMARK 2.2. The definitions of the $\alpha$ order Caputo fractional derivatives require that $f^{(n)}$ exists almost everywhere. The relationship between Riemann-Liouville and Caputo fractional derivatives is given by the following identities:

$$
\begin{aligned}
& { }_{a}^{C} D_{x}^{\alpha} f(x):={ }_{a} D_{x}^{\alpha} f(x)-\sum_{k=0}^{n-1} \frac{f^{(k)}(a)}{\Gamma(k+1-\alpha)}(x-a)^{k-\alpha}, \\
& { }_{x}^{C} D_{b}^{\alpha} f(x):={ }_{x} D_{b}^{\alpha} f(x)-\sum_{k=0}^{n-1} \frac{f^{(k)}(b)}{\Gamma(k+1-\alpha)}(b-x)^{k-\alpha} .
\end{aligned}
$$

It is easy to check that the following "weak" definitions of the Caputo fractional derivatives in the case $0<\alpha<1$ :

$$
\begin{aligned}
& { }_{a}^{C} D_{x}^{\alpha} f(x):={ }_{a} D_{x}^{\alpha}[f(x)-f(a)]=\frac{d}{d x}\left[{ }_{a} I_{x}^{1-\alpha}(f(x)-f(a))\right], \\
& { }_{x}^{C} D_{b}^{\alpha} f(x):={ }_{x} D_{b}^{\alpha}[f(x)-f(b)]=-\frac{d}{d x}\left[{ }_{x} I_{b}^{1-\alpha}(f(x)-f(b))\right],
\end{aligned}
$$

which do not require the existence of $f^{\prime}(x)$, instead, they require the existence of $f(a)$ and $f(b)$, respectively.

Unlike the Riemann-Liouville and Caputo derivatives which use an integral operator to induce a fractional order derivative, a natural question is if a fractional derivative can be defined as a limit of some difference quotient similar to the definition of the integer order derivative. Although there have been some attempts in this direction (cf. [17]), we only recall the well-known Grünwald-Letnikov fractional derivatives as they are related to the Riemann-Liouville derivatives.

Definition 2.4 (cf. 30]). Let $0<\alpha<1$ and $f:[a, b] \rightarrow \mathbb{R}$. The left GrünwaldLetnikov fractional derivative of $f$ is defined by

$$
{ }_{a}^{G L} D_{x}^{\alpha} f(x):=\lim _{h \rightarrow 0^{+}} \frac{1}{h^{\alpha}} \sum_{k=0}^{[(x-a) / h]} \frac{(-1)^{k} \Gamma(1+\alpha)}{\Gamma(k+1) \Gamma(\alpha-k+1)} f(x-k h) \quad \forall x \in[a, b]
$$


and the right Grünwald-Letnikov fractional derivative of $f$ is defined by

$$
{ }_{x}^{G L} D_{b}^{\alpha} f(x):=\lim _{h \rightarrow 0^{+}} \frac{1}{h^{\alpha}} \sum_{k=0}^{[(b-x) / h]} \frac{(-1)^{k+1} \Gamma(1+\alpha)}{\Gamma(k+1) \Gamma(\alpha-k+1)} f(x+k h) \quad \forall x \in[a, b] .
$$

Clearly, for the fractional derivative, the difference quotients are much more complicated. It can be shown [30] that the Grünwald-Letnikov fractional derivative and the Riemann-Liouville derivative are equivalent for sufficient smooth functions.

2.1.2. Definitions on an Infinite Interval. The fractional integrals over unbounded intervals are defined in the same way; here we only consider the whole real line case, that is, $(a, b)=(-\infty, \infty)$. There are two different definitions of fractional order derivatives in the infinite interval case which were proved to be equivalent. The first three definitions are direct generalizations of Definitions 2.1 2.3 ,

Definition 2.5 (cf. [30]). Let $\sigma>0$ and $f: \mathbb{R} \rightarrow \mathbb{R}$. The $\sigma$ order left Liouville fractional integral of $f$ is defined by

$$
I_{x}^{\sigma} f(x):=\frac{1}{\Gamma(\sigma)} \int_{-\infty}^{x} \frac{f(y)}{(x-y)^{1-\sigma}} d y \quad \forall x \in \mathbb{R}
$$

and the $\sigma$ order right Liouville fractional integral of $f$ is defined by

$$
{ }_{x} I^{\sigma} f(x):=\frac{1}{\Gamma(\sigma)} \int_{x}^{\infty} \frac{f(y)}{(y-x)^{1-\sigma}} d y \quad \forall x \in \mathbb{R} .
$$

Definition 2.6 (cf. [30]). Let $n-1<\alpha<n$ and $f: \mathbb{R} \rightarrow \mathbb{R}$. The $\alpha$ order left Liouville fractional derivative of $f$ is defined by

$$
D_{x}^{\alpha} f(x):=\frac{1}{\Gamma(n-\alpha)} \frac{d^{n}}{d x^{n}} \int_{-\infty}^{x} \frac{f(y)}{(x-y)^{1+\alpha-n}} d y \quad \forall x \in \mathbb{R}
$$

and the $\alpha$ order right Liouville fractional derivative of $f$ is defined by

$$
{ }_{x} D^{\alpha} f(x):=\frac{(-1)^{n}}{\Gamma(n-\alpha)} \frac{d^{n}}{d x^{n}} \int_{x}^{\infty} \frac{f(y)}{(y-x)^{1+\alpha-n}} d y \forall x \in \mathbb{R} .
$$

Definition 2.7 (cf. [30]). Let $n-1<\alpha<n$ and $f: \mathbb{R} \rightarrow \mathbb{R}$. The $\alpha$ order left Caputo fractional derivative of $f$ is defined by

$$
{ }^{C} D_{x}^{\alpha} f(x):=\frac{1}{\Gamma(n-\alpha)} \int_{-\infty}^{x} \frac{f^{(n)}(y)}{(x-y)^{1+\alpha-n}} d y \quad \forall x \in \mathbb{R}
$$

and the $\alpha$ order right Caputo fractional derivative of $f$ is defined by

$$
{ }_{x}^{C} D^{\alpha} f(x):=\frac{(-1)^{n}}{\Gamma(n-\alpha)} \int_{x}^{\infty} \frac{f^{(n)}(y)}{(y-x)^{1+\alpha-n}} d y \quad \forall x \in \mathbb{R} .
$$

It should also be noted that all integrals over the infinite domain are understood as standard improper integrals. 
Similar to the finite interval case, we also can define the Grünwald-Letnikov fractional derivatives for functions defined on the whole real line. In this case, notice that the sums are infinite sums in the above definition.

Definition 2.8 (cf. [30]). Let $0<\alpha<1$ and $f: \mathbb{R} \rightarrow \mathbb{R}$. The left GrünwaldLetnikov fractional derivative of $f$ is defined by

$$
{ }^{G L} D_{x}^{\alpha} f(x):=\lim _{h \rightarrow 0^{+}} \frac{1}{h^{\alpha}} \sum_{k=0}^{\infty} \frac{(-1)^{k} \Gamma(1+\alpha)}{\Gamma(k+1) \Gamma(\alpha-k+1)} f(x-k h) \quad \forall x \in \mathbb{R}
$$

and the right Grünwald-Letnikov fractional derivative of $f$ is defined by

$$
{ }_{x}^{G L} D^{\alpha} f(x):=\lim _{h \rightarrow 0^{+}} \frac{1}{h^{\alpha}} \sum_{k=0}^{\infty} \frac{(-1)^{k+1} \Gamma(1+\alpha)}{\Gamma(k+1) \Gamma(\alpha-k+1)} f(x+k h) \quad \forall x \in \mathbb{R} .
$$

Next, we recall another definition of fractional derivatives that are based on the Fourier transforms.

Definition 2.9 (cf. [30]). Let $\alpha>0$ and $f: \mathbb{R} \rightarrow \mathbb{R}$. The $\alpha$ order Fourier fractional derivative is defined by

$$
{ }^{\mathcal{F}} D^{\alpha} f(x):=\mathcal{F}^{-1}\left[(i \xi)^{\alpha} \mathcal{F}[f](\xi)\right](x) \quad \forall x \in \mathbb{R}
$$

where $\mathcal{F}[\cdot]$ and $\mathcal{F}^{-1}[\cdot]$ denote respectively the Fourier transform and its inverse transform which are defined as follows: for any $x, \xi \in \mathbb{R}$

$$
\mathcal{F}[f](\xi):=\int_{\mathbb{R}} e^{-i \xi x} f(x) d x, \quad \mathcal{F}^{-1}[f](x):=\int_{\mathbb{R}} e^{i \xi x} f(\xi) d \xi .
$$

REMARK 2.3. The above Fourier fractional order derivative notion is based on the following well-known property of the Fourier transform:

$$
\mathcal{F}\left[f^{(n)}\right](\xi)=(i \xi)^{n} \mathcal{F}[f](\xi), \quad f^{(n)}(x)=\mathcal{F}^{-1}\left[(i \xi)^{n} \mathcal{F}[f]\right](x)
$$

for any positive integer $n$.

2.2. Action on Smooth Functions with Compact Support. The action of the Riemann-Liouville integral and differential operators on smooth functions with compact support is of special interest for our study in this paper. The need for understanding these behaviors will become evident in the later sections.

We now have a closer look at the support and the tail behavior of ${ }^{ \pm} D^{\alpha} \varphi$ for $\varphi \in C_{0}^{\infty}(\mathbb{R})$ so that $\operatorname{supp}(\varphi) \subset(a, b)$. To that end, a direct computation yields

$$
{ }^{-} I_{x}^{\sigma} \varphi(x)= \begin{cases}0 & \text { if } x \in(-\infty, a) \\ { }_{a} I_{x}^{\sigma} \varphi(x) & \text { if } x \in[a, b] \\ L(x) & \text { if } x \in(b, \infty)\end{cases}
$$

where

$$
L(x)=\frac{1}{\Gamma(\sigma)} \int_{a}^{b} \frac{\varphi(y)}{(x-y)^{1-\sigma}} d y
$$


Taking the first derivative and letting $\sigma=1-\alpha$ yields

$$
D_{x}^{\alpha} \varphi(x)= \begin{cases}0 & \text { if } x \in(-\infty, a), \\ { }_{a} D_{x}^{\alpha} \varphi(x) & \text { if } x \in[a, b], \\ L^{\prime}(x) & \text { if } x \in(b, \infty),\end{cases}
$$

A similar formula can be shown for the right direction. The pollution function will be denoted by $R(x)$ corresponding to $L(x)$.

Proposition 2.10. If $\varphi \in C_{0}^{\infty}(\mathbb{R})$ with $\operatorname{supp}(\varphi) \subset(a, b)$, then $\operatorname{supp}\left(D_{x}^{\alpha} \varphi\right) \subset$ $(a, \infty)$ and $\operatorname{supp}\left({ }_{x} D^{\alpha} \varphi\right) \subset(-\infty, b)$.

REMARK 2.4. (a) Riemann-Liouville fractional differential (and integral) operators have a pollution effect on the support when acting on functions in $C_{0}^{\infty}(\Omega)$. Left derivatives pollute the support to the right and right derivatives pollute the support to the left. This pollution effect is a consequence of the nonlocal characteristics of fractional order differential and integral operators; in particular, the "memory" effect.

(b) When $x \rightarrow \pm \infty$, the integrands in $L^{\prime}(x)$ and $R^{\prime}(x)$ are shrinking. Moreover, $\lim _{x \rightarrow \infty}\left|L^{\prime}(x)\right|=0$ (and $\lim _{x \rightarrow-\infty}\left|R^{\prime}(x)\right|=0$ ).

The next theorem states an integrability property of $D^{\alpha} \varphi$ for $\varphi \in C_{0}^{\infty}(\mathbb{R})$.

Theorem 2.11. Let $0<\alpha<1$. If $\varphi \in C_{0}^{\infty}(\Omega)$, then ${ }^{ \pm} D^{\alpha} \varphi \in L^{p}(\Omega)$ for each $1 \leq p \leq \infty$

Proof. The proof comes from direct calculations that can be found in 9 .

REMARK 2.5. A special class of compactly supported smooth functions are those obtained through a mollification process (i.e., through a convolution with a compactly supported mollifier). We refer the reader to [9] for a detailed discussion.

2.3. Fundamental Theorem of Classical Fractional Calculus (FTcFC). In this subsection we present an alternative understanding of the classical fractional order integrals and derivatives. That is to interpret the fractional differentiation as a by-product of the fractional integration through the so-called Fundamental Theorem of Fractional Calculus. This new interpretation will play an important role in the development of our weak fractional calculus theory to be given in the next section.

2.3.1. FTcFC on Finite Intervals $(a, b) \subset \mathbb{R}$. We begin this subsection by recalling the following properties of the fractional operators ${ }^{ \pm} I^{\alpha}$ and ${ }^{ \pm} D^{\alpha}$.

LEMma 2.12 (cf. 30). Let $0<\alpha<1$. The following properties hold:

(a) ${ }_{a} D_{x}^{\alpha} \kappa_{-}^{\alpha}(x) \equiv 0$ and ${ }_{x} D_{b}^{\alpha} \kappa_{+}^{\alpha}(x) \equiv 0$ where

$$
\kappa_{-}^{\alpha}(x):=(x-a)^{\alpha-1}, \quad \kappa_{+}^{\alpha}(x):=(b-x)^{\alpha-1} .
$$

(b) ${ }_{a} D_{x}^{\alpha}{ }_{a}^{\alpha} I_{x}^{\alpha} f(x)=f(x)$ and ${ }_{x} D_{b x}^{\alpha} I_{b}^{\alpha} f(x)=f(x)$ for any $f \in L_{l o c}^{1}((a, b))$.

(c) If $I_{x}^{1-\alpha} f \in A C([a, b])$, then

$$
f(x)=c_{-}^{1-\alpha} \kappa_{-}^{\alpha}(x)+{ }_{a} I_{x}^{\alpha} a D_{x}^{\alpha} f(x),
$$

and if $I_{x}^{1-\alpha} f \in A C([a, b])$, then

$$
f(x)=c_{+}^{1-\alpha} \kappa_{+}^{\alpha}(x)+{ }_{x} I_{b x}^{\alpha} D_{b}^{\alpha} f(x),
$$

where

$$
c_{-}^{\sigma}:=\frac{{ }_{a} I_{x}^{\sigma} f(a)}{\Gamma(\sigma)}, \quad c_{+}^{\sigma}:=\frac{{ }_{x} I_{b}^{\sigma} f(b)}{\Gamma(\sigma)}
$$


On noting the fact that ${ }^{ \pm} D^{\alpha} F(x)=f(x)$ implies that ${ }^{ \pm} I^{1-\alpha} F \in A C([a, b])$, then the above lemma immediately infers the following theorem.

Theorem 2.13. Let $0<\alpha<1, f, F \in L^{1}((a, b))$. Then ${ }^{ \pm} D^{\alpha} F(x)=f(x)$ on $(a, b)$ if and only if

$$
F(x)=c_{ \pm}^{1-\alpha} \kappa_{ \pm}^{\alpha}(x)+{ }^{ \pm} I^{\alpha} f(x) .
$$

REMARK 2.6. The analogue of Theorem 2.13 in the integer order calculus is the well-known Fundamental Theorem of Calculus (or Newton-Leibniz Theorem) which says that $F^{\prime}(x):=\frac{d F}{d x}(x)=f(x)$ if and only if

$$
F(x)=F(a)+\int_{a}^{x} f(y) d y=F(a)+\int_{a}^{b} H(x-y) f(y) d y \quad \forall x \in[a, b],
$$

where the kernel function $\kappa(x, y)=H(x-y)$, the Heaviside function. Since the kernel space of the derivative $\frac{d}{d x}$ operator is $\mathbb{R}$, this is why the first term on the right-hand side must be a constant, because it must belong to the kernel space of $\frac{d}{d x}$. Due to the above analogue, we shall call Theorem 2.13 Fundamental Theorem of Classical Fractional Calculus on finite intervals in the rest of this paper.

In fact, given integral operators ${ }^{ \pm} I^{\alpha},(2.18)$ can be used to define the corresponding Riemann-Liouville derivatives as follows.

DeFinition 2.14. Let $0<\alpha<1$ and $f, F \in L^{1}((a, b))$. Then $f$ is called the $\alpha$ order left/right Riemann-Liouville fractional derivative of $F$, and write ${ }^{ \pm} D^{\alpha} F(x)=$ $f(x)$, if (2.18) holds,

$$
F(x)=c_{ \pm}^{1-\alpha} \kappa^{ \pm}(x)+{ }^{ \pm} I^{\alpha} f(x) .
$$

It is easy to check that the $\alpha$ order fractional derivative of $f$, if it exists, is uniquely defined. In light of Theorem 2.13, we see that the original definition and the above definition are equivalent. In this paper we emphasize the above FTFC approach of using a given integral operator (i.e., its kernel function is given) to define the corresponding derivative notion by the FTFC identity. There are many benefits/advantages of this approach. It is systematic (not ad hoc) and quite general, because it is done in the same way for any given integral operator (see the definition below). The FTFC is built into the definition; we regard that having such a FTFC is essential for any fractional calculus theory.

We now give the alluded definition of fractional derivatives for general kernels (and their associated integral operators).

Definition 2.15. Given any kernel function $\tau \in L^{1}((a, b) \times(a, b))$, let $I_{\tau}$ denote the subordinate (Riemann or Lebesgue) integral operator, namely,

$$
I_{\tau} f(x):=\int_{a}^{b} \tau(x, y) f(y) d y \quad \forall x \in[a, b] .
$$

Let $f, F \in L^{1}(\Omega)$, then $f$ is called the fractional/nonlocal derivative of $F$, and written $D_{\tau} F=f$, there exists some $c \in[a, b]$ such that

$$
F(x)=C_{F, c} \tau(x, c)+I_{\tau} f(x) \quad \forall x \in[a, b]
$$

for some constant $C_{F, c}$ depending on both $F$ and $c$. 
2.3.2. FTcFC on the Infinite Interval $\mathbb{R}$. The case for a FTcFC on the entire line is quite different, but simpler because of the decay properties of kernel functions $\kappa_{ \pm}^{\alpha}$ when $|x| \rightarrow \infty$. Similarly, we start by recalling the following properties of the fractional operators ${ }^{ \pm} I^{\alpha}$ and ${ }^{ \pm} D^{\alpha}$.

Lemma 2.16 (cf. 30]). Let $0<\alpha<1$. The following properties hold:

(a) $D_{x}^{\alpha} I_{x}^{\alpha} f(x)=f(x)$ and ${ }_{x} D^{\alpha}{ }_{x} I^{\alpha} f(x)=f(x)$ for any $f \in L^{1}(\mathbb{R})$,

(b) $I_{x}^{\alpha} D_{x}^{\alpha} f(x)=f(x)$ and ${ }_{x} I^{\alpha}{ }_{x} D^{\alpha} f(x)=f(x)$ for any $I^{1-\alpha} f \in A C(\mathbb{R})$ so that $f(x) \rightarrow 0$ as $|x| \rightarrow \infty$.

We then have

Theorem 2.17. Let $0<\alpha<1$, and $f, F \in L^{1}(\mathbb{R})$. If

$$
F(x)={ }^{ \pm} I^{\alpha} f(x),
$$

then ${ }^{ \pm} D^{\alpha} F(x)=f(x)$. The converse is also true if $F(x) \rightarrow 0$ as $|x| \rightarrow \infty$ is required.

For the same reason as given in Subsection 2.3.1, we shall call Theorem 2.17 the Fundamental Theorem of Classical Fractional Calculus on $\mathbb{R}$ in the rest of this paper.

Similarly, we also introduce the following definition.

Definition 2.18. Let $0<\alpha<1$ and $f, F \in L^{1}(\mathbb{R})$. Then $f$ is called the $\alpha$ order left/right Riemann-Liouville fractional derivative of $F$ on $\mathbb{R}$, and write ${ }^{ \pm} D^{\alpha} F(x)=$ $f(x)$ (abusing the notation), if (2.22) holds.

It is easy to show that ${ }^{ \pm} D^{\alpha} F$ is well defined and it coincides with the original definitions of Riemann-Liouville derivatives on $\mathbb{R}$. This FTcFC interpretation of fractional derivatives will be emphasized in this paper.

3. A Weak Fractional Differential Calculus Theory. We saw in the previous section that the classical fractional calculus theory has several difficulties arising from the change to non-integer order integration and differentiation. Unlike the well formulated and understood integer order calculus, the basic notion of fractional derivatives is domain-dependent and has several different (and nonequivalent) definitions; familiar calculus rules do not hold or become fairly complicated and restricted; fractionally differentiable functions are difficult to characterize; there is no local characterization of non-local fractional integral and derivative operators; more importantly, although the Riemann integration can be generalized to the Lebesgue integration in the definitions of all fractional integrals, unlike the integer order case, there is no weak fractional derivative concept/theory, which in turn has caused some difficulties and confusions for studying/interpreting fractional order differential equations.

The primary goal of this section (and this paper) is to develop a weak fractional differential calculus theory, which is parallel to the integer order weak derivative theory (cf. [1, 3, 8]). The anticipated weak fractional theory lays down the ground work for developing a new fractional Sobolev space theory in a companion paper [10]. Together they will provide a solid theoretical foundation and pave the way for a systematic and thorough study of initial value, boundary value and initial-boundary value problems for fractional order differential equations and fractional calculus of variations problems as well as their numerical solutions in the subsequent works [11, 12.

In this section, unless it is stated otherwise, all integrals are understood in the Lebesgue sense. We use ${ }^{-} D^{\alpha}$ and ${ }^{+} D^{\alpha}$ to denote respectively any left and right $\alpha$ order classical derivative introduced in Section $2,{ }^{ \pm} D^{\alpha}$ denotes either ${ }^{-} D^{\alpha}$ or ${ }^{+} D^{\alpha}$. $\Omega$ denotes either a finite interval $(a, b)$ or the whole real line $\mathbb{R}$. In the case $\Omega=(a, b)$, for any $\varphi \in C_{0}^{\infty}(\Omega), \tilde{\varphi}$ is used to denote the zero extension of $\varphi$ to $\mathbb{R}$. 
3.1. Definitions of Weak Fractional Derivatives. Like in the integer order case, the idea of defining weak fractional derivative ${ }^{ \pm} \mathcal{D}^{\alpha} u$ of a function $u$ is to specify its action on any smooth compactly supported function $\varphi \in C_{0}^{\infty}(\Omega)$, instead of knowing its pointwise values as done in the classical fractional derivative definitions.

Definition 3.1. For $\alpha>0$, let $[\alpha]$ denote the integer part of $\alpha$. For $u \in L^{1}(\Omega)$,

(i) a function $v \in L_{l o c}^{1}(\Omega)$ is called the left weak fractional derivative of $u$ if

$$
\int_{\Omega} v(x) \varphi(x) d x=(-1)^{[\alpha]} \int_{\Omega} u(x)^{+} D^{\alpha} \tilde{\varphi}(x) d x \quad \forall \varphi \in C_{0}^{\infty}(\Omega),
$$

we write ${ }^{-} \mathcal{D}^{\alpha} u:=v$;

(ii) a function $w \in L_{l o c}^{1}(\Omega)$ is called the right weak fractional derivative of $u$ if

$$
\int_{\Omega} w(x) \varphi(x) d x=(-1)^{[\alpha]} \int_{\Omega} u(x)^{-} D^{\alpha} \tilde{\varphi}(x) d x \quad \forall \varphi \in C_{0}^{\infty}(\Omega),
$$

and we write ${ }^{+} \mathcal{D}^{\alpha} u:=w$.

The next proposition shows that weak fractional derivatives are well-defined.

Proposition 3.2. Let $u \in L^{1}(\Omega)$. Then a weak fractional derivative of $u$, if it exists, is uniquely defined. $u$, then

Proof. Let $v_{1}, v_{2} \in L_{l o c}^{1}(\Omega)$ be two left (resp. right) weak fractional derivatives of

$$
\int_{\Omega} v_{1}(x) \varphi(x) d x=(-1)^{[\alpha]} \int_{\Omega} u(x)^{ \pm} D^{\alpha} \tilde{\varphi}(x) d x=\int_{\Omega} v_{2}(x) \varphi(x) d x \quad \forall \varphi \in C_{0}^{\infty}(\Omega) .
$$

Thus,

$$
0=\int_{\Omega}\left(v_{1}(x)-v_{2}(x)\right) \varphi(x) d x \quad \forall \varphi \in C_{0}^{\infty}(\Omega) .
$$

Therefore, $v_{1}=v_{2}$ almost everywhere. The proof is complete.

A few remarks are given below to help understand the above definition.

REMARK 3.1. (a) The introduction of $\tilde{\varphi}$ in the definitions makes the weak fractional derivatives intrinsic in the sense that ${ }^{ \pm} D^{\alpha} \tilde{\varphi}$ is independent of the choice of ${ }^{ \pm} D^{\alpha}$, because $D_{x}^{\alpha} \tilde{\varphi}={ }_{a} D_{x}^{\alpha} \tilde{\varphi}={ }^{\mathcal{F}} D^{\alpha} \tilde{\varphi}$ and ${ }_{x} D^{\alpha} \tilde{\varphi}={ }_{x} D_{b}^{\alpha} \tilde{\varphi}={ }^{\mathcal{F}} D^{\alpha} \tilde{\varphi}$.

(b) The constant $(-1)^{[\alpha]}$ helps guarantee consistency with the integer order case.

(c) Integration by parts is built into the definitions.

(d) The reason to require $u \in L^{1}(\Omega)$ is because ${ }^{ \pm} D^{\alpha} \tilde{\varphi} \in L^{\infty}(\mathbb{R})$ is not compactly supported. When $\alpha \in \mathbb{N}$, this condition can be relaxed to $L_{l o c}^{1}(\Omega)$. In fact, the restriction $u \in L^{1}(\Omega)$ can be relaxed to the weighted $L^{1}$ space $u \in L^{1}(\Omega, \rho)$ with the weight $\rho=L^{\prime}$ or $\rho=R^{\prime}$.

(e) As expected, weak fractional derivatives are domain-dependent. However, unlike the classical fractional derivatives, whose domain dependence is explicitly shown in the limits of the integrals involved, the domain dependence of weak fractional derivatives is implicitly introduced by using domain-dependent test functions $\varphi \in C_{0}^{\infty}(\Omega)$.

(f) The above definitions can be easily extended to non-interval domains or subdomains of $\Omega$. Indeed, given a bounded set $E \subset \mathbb{R}$, the only changes which need to be made in the definitions are to replace $\Omega$ by $E$ and $\varphi \in C_{0}^{\infty}(\Omega)$ by $\varphi \in C_{0}^{\infty}\left(\left(a^{*}, b^{*}\right)\right)$ where $\left(a^{*}, b^{*}\right)=\cap\{(c, d): E \subset(c, d)\}$, the smallest interval which contains $E$.

(g) Extensions of the above definitions to distributions will be given in Section 4 . 
The following result is trivial and expected, see [9, Theorem 2.5] for a proof.

Proposition 3.3. Let $u$ be Riemann-Liouville differentiable such that ${ }^{ \pm} D^{\alpha} u \in$ $L_{\text {loc }}^{1}(\Omega)$. Then ${ }^{ \pm} \mathcal{D}^{\alpha} u={ }^{ \pm} D^{\alpha} u$ almost everywhere.

The next result shows the consistency with integer order weak derivatives.

Proposition 3.4. Let $n-1<\alpha<n$. The $\alpha$ order weak fractional derivative converges to the $n^{\text {th }}$ order weak derivative almost everywhere as $\alpha \rightarrow n$.

Proof. Consider the case when $n=1$; the others follow similarly. In order to prove that ${ }^{ \pm} \mathcal{D}^{\alpha} u \rightarrow \mathcal{D} u$ almost everywhere as $\alpha \rightarrow 1$, we see that

$$
\begin{aligned}
0 & =\int_{\Omega} u \varphi^{\prime} d x+\lim _{\alpha \rightarrow 1}(-1)^{[\alpha]} \int_{\Omega} u^{\mp} D^{\alpha} \varphi d x \\
& =\lim _{\alpha \rightarrow 1} \int_{\Omega}{ }^{ \pm} \mathcal{D}^{\alpha} u \varphi d x-\int_{\Omega} \mathcal{D} u \cdot \varphi d x \\
& =\lim _{\alpha \rightarrow 1} \int_{\Omega}\left({ }^{ \pm} \mathcal{D}^{\alpha} u-\mathcal{D} u\right) \varphi d x
\end{aligned}
$$

which follows by the consistency of classical derivatives on functions $\varphi \in C_{0}^{\infty}(\Omega)$.

3.2. Relationships with Other Derivative Notions. Although the notion of a weak fractional derivative is analogous to the integer order weak derivative and hence is deserving of the name in this sense, we provide simple examples to illustrate the following points.

(a) The notion of a weak fractional derivative is a unifying concept of fractional differentiation with respect to the derivatives defined in Section 2

(b) Weak fractional derivatives can exist for functions whose classical fractional derivatives do not exist.

(c) Functions that do not have first order weak derivatives may have weak fractional derivatives.

First, we give a simple example to demonstrate that the weak fractional derivative is a unifying concept (item (a) above). To illustrate this point, we consider $\Omega=\mathbb{R}$, $0<\alpha<1$, and $c \in \mathbb{R} \backslash\{0\}$, let $u(x) \equiv c$. Trivially,

$$
{ }^{C} D_{x}^{\alpha} u(x)=\frac{1}{\Gamma(1-\alpha)} \int_{-\infty}^{x} \frac{u^{\prime}(y)}{(x-y)^{-\alpha}} d y=0,
$$

hence, the Caputo derivative is identically zero. However,

$$
D_{x}^{\alpha} u(x)=\frac{1}{\Gamma(1-\alpha)} \frac{d}{d x} \int_{-\infty}^{x} \frac{c}{(x-y)^{\alpha}} d y=\frac{c}{\Gamma(1-\alpha)} \frac{d}{d x}\left(\left.\frac{(x-y)^{1-\alpha}}{1-\alpha}\right|_{y=x} ^{y=-\infty}\right)
$$

which does not exist as a function because the singular integral diverges, hence, the Riemann-Liouville fractional derivative does not exist on $\mathbb{R}$. Thus in the classical case, the choice of fractional derivative definition becomes essential.

We now compute the weak fractional derivative of $u$ below. For any $\varphi \in C_{0}^{\infty}(\mathbb{R})$,

$$
\int_{\mathbb{R}} c^{+} D^{\alpha} \varphi(x) d x=\int_{\mathbb{R}} c \frac{d}{d x} I_{x}^{1-\alpha} \varphi(x) d x=\left.c\left[I_{x}^{1-\alpha} \varphi(x)\right]\right|_{-\infty} ^{\infty}=0 .
$$

Therefore, the weak derivative exists and is equal to zero, which coincides with the Caputo derivative. Here we see that by forcing the integration by parts formula to hold, the definition automatically selects the appropriate fractional derivative. 
What if $\Omega=(a, b)$ is finite? In this case, we know that $0={ }_{a}^{C} D_{x}^{\alpha} c \neq{ }_{a} D_{x}^{\alpha} c=$ $c \Gamma(1-\alpha)^{-1}(x-a)^{-\alpha}$. A simple calculation yields that

$$
\int_{a}^{b} c^{+} D^{\alpha} \varphi(x) d x=c \int_{a}^{b} \frac{d}{d x}{ }_{x} I_{b}^{\alpha} \varphi(x) d x=\left.c\left[{ }_{x} I_{b}^{\alpha} \varphi(x)\right]\right|_{a} ^{b}=c_{a} I_{b}^{\alpha} \varphi(b)
$$

holds for all $\varphi \in C_{0}^{\infty}((a, b))$, which shows that the Caputo derivative of constant $c$ (that is zero) can not satisfy the integration by parts formula. Hence, ${ }_{a}^{C} D_{x}^{\alpha} c \neq^{-} \mathcal{D}^{\alpha} c$. However, a direct computation shows that

$$
\int_{a}^{b} c^{+} D^{\alpha} \varphi d x=\int_{a}^{b} \varphi_{a} D_{x}^{\alpha} c d x \quad \forall \varphi \in C_{0}^{\infty}((a, b)) .
$$

Hence, ${ }^{-} \mathcal{D}^{\alpha} c={ }_{a} D_{x}^{\alpha} c$. Again, we see that the built-in feature of an integration by parts formula effectively selects an appropriate fractional derivative.

Next, we illustrate that the notion of weak fractional derivatives is truly a generalization of the notion of classical fractional derivatives by showing that there are functions whose weak derivatives exist, but classical fractional (Rieamann-Liouville) derivatives do not. Moreover, we give a characterization of functions that are weakly differentiable, which parallels the characterization for first order weakly differentiable functions. In lieu of concrete examples, we demonstrate that there is a procedural way to produce functions that are not Riemann-Liouville differentiable, but are weakly differentiable. Notice that for $u \in L^{1}(\Omega)$ and $\varphi \in C_{0}^{\infty}(\Omega)$, there holds

$$
\int_{\Omega} u^{\mp} D^{\alpha} \varphi d x=\int_{\Omega} u^{\mp} I^{1-\alpha} \varphi^{\prime} d x=\int_{\Omega}{ }^{ \pm} I^{1-\alpha} u \varphi^{\prime} d x .
$$

In order to perform an integration by parts on the right side, we need that ${ }^{ \pm} I^{1-\alpha} u \in$ $W^{1,1}(\Omega)$ (or at least absolutely continuous). In that case, the function $u$ then has a weak fractional derivative. On the other hand, we want the function $u$ not to be Riemann-Liouville differentiable, which requires that ${ }^{ \pm} I^{1-\alpha} u \notin C^{1}(\Omega)$. Since ${ }^{ \pm} I^{1-\alpha} u \in W^{1,1}(\Omega)$ does not imply ${ }^{ \pm} I^{1-\alpha} u \in C^{1}(\Omega)$, then we want to find $u \in L^{1}(\Omega)$ so that ${ }^{ \pm} I^{1-\alpha} u=f$ for a given function $f \in W^{1,1}(\Omega)$, but $f \notin C^{1}(\Omega)$. There are many such $f$ functions, the best known example perhaps is $f(x)=|x|$.

It follows from Lemma 2.12 that we obtain the desired examples by taking $u={ }^{ \pm} D^{1-\alpha} f$ for any $f \in\left\{W^{1,1}(\Omega) ; f \notin C^{1}(\Omega)\right.$ and ${ }^{ \pm} D^{1-\alpha} f$ exists $\}$. By the characterization of functions in $W^{1,1}(\Omega)$, we conclude that $u$ is weakly fractional differentiable with ${ }^{ \pm} \mathcal{D}^{\alpha} u \in L^{1}(\Omega)$ if and only ${ }^{ \pm} I^{1-\alpha} u$ is absolutely continuous.

REMARK 3.2. The above procedure can be relaxed to characterize all weakly fractional differentiable functions by requiring $f$ to be only first order weakly differentiable; rather than $f \in W^{1,1}(\Omega)$. However, the above procedure does produce a rich (and nearly complete) validation of item (b) above.

Finally, we compare the weak fractional derivative to the integer order weak derivative; in particular, we demonstrate that the notion of weak fractional derivative is indeed consistent with, and extends, the notion of integer order weak derivatives by identifying a class of functions so that their weak fractional derivatives exist, but their integer order weak derivatives do not.

To that end, consider $\Omega=(-1,1)$ and $\lambda, \mu \in \mathbb{R}$ so that $\lambda \neq \mu$, then define

$$
u(x):= \begin{cases}\lambda & \text { if }-1<x<0 \\ \mu & \text { if } 0<x<1\end{cases}
$$


a genuine step function. Let $\mathcal{D}$ denote the first order weak derivative operator. Obviously, $\mathcal{D} u$ does not exist (cf. [3]) because $u \notin C((-1,1)$ ); such a function has only a distributional derivative. However, a direct calculation shows that

$$
\int_{-1}^{1} u^{\mp} D^{\alpha} \varphi d x=\int_{-1}^{1} \varphi^{ \pm} \mathcal{D}^{\alpha} u d x \quad \forall \varphi \in C_{0}^{\infty}((-1,1))
$$

holds, where

$$
{ }^{-} \mathcal{D}^{\alpha} u(x):= \begin{cases}\frac{1}{\Gamma(1-\alpha)} \frac{\lambda}{(x+1)^{\alpha}} & \text { if } x \in(-1,0], \\ \frac{1}{\Gamma(1-\alpha)}\left(\frac{\lambda}{(x+1)^{\alpha}}-\frac{\lambda}{x^{\alpha}}+\frac{\mu}{x^{\alpha}}\right) & \text { if } x \in(0,1) .\end{cases}
$$

A similar formula also holds for ${ }^{+} \mathcal{D}^{\alpha}$. Note that the weak derivative is locally integrable. In fact, since $0<\alpha<1$, it is globally integrable; an observation that is foundational to density properties in the fractional Sobolev spaces introduced in 10. Thus, we have shown that all step functions are weakly fractional differentiable, but are not weakly differentiable to any integer order. In fact, it can be shown that the same conclusion also holds for all piecewise smooth, but globally discontinuous functions. Simple exams are given in [3, 8].

3.3. Approximation and Characterization of Weak Fractional Derivatives. In this subsection we present a characterization for weak fractional derivatives so that they can be approached/understood from a different, but equivalent point of view. Like in the integer order case, we prove that weakly fractional differentiable functions can be approximated by smooth functions. Unless it is stated otherwise, we assume $0<\alpha<1$ in this subsection.

3.3.1. The Finite Interval Case. We first consider the case when $\Omega:=(a, b) \subset$ $\mathbb{R}$ is a finite interval. Let $\varepsilon>0$, define the $\varepsilon$ - interior of $\Omega$ as $\Omega_{\varepsilon}:=\{x \in \Omega$ : $\operatorname{dist}(x, \partial \Omega)>\varepsilon\}$.

Lemma 3.5. Suppose ${ }^{ \pm} \mathcal{D}^{\alpha} u \in L_{l o c}^{1}(\Omega)$ exists. Then

$$
{ }^{ \pm} \mathcal{D}^{\alpha} \tilde{u}^{\varepsilon}=\eta_{\varepsilon}{ }^{ \pm} \mathcal{D}^{\alpha} u \quad \text { a.e. in } \Omega_{\varepsilon}
$$

where $\eta_{\varepsilon}$ denotes the standard mollifier and $\tilde{u}^{\varepsilon}$ stands for the mollification of $\tilde{u}$.

We omit the proof to save space and refer the reader to [9, Lemma 3.3] for details.

The next theorem gives a characterization of fractional order weak derivatives.

TheOREm 3.6. Let $u \in L^{1}(\Omega)$. Then $v={ }^{ \pm} \mathcal{D}^{\alpha} u$ in $L_{\text {loc }}^{1}(\Omega)$ if and only if there exists a sequence $\left\{u_{j}\right\}_{j=1}^{\infty} \subset C^{\infty}(\Omega)$ such that $u_{j} \rightarrow u$ in $L^{1}(\Omega)$ and ${ }^{ \pm} \mathcal{D}^{\alpha} u_{j} \rightarrow v$ in $L_{l o c}^{1}(\Omega)$ as $j \rightarrow \infty$.

Proof. Let $u \in L^{1}(\Omega)$ and $u^{\varepsilon}$ denote its mollification.

Step 1: Suppose that $v={ }^{ \pm} \mathcal{D}^{\alpha} u \in L_{l o c}^{1}(\Omega)$. Let $\tilde{u}^{\varepsilon}$ denote the mollification of $\tilde{u}$. By the properties of mollification, $\tilde{u}^{\varepsilon} \rightarrow u$ in $L^{1}(\Omega)$ as $\varepsilon \rightarrow 0$. From lemma, we have ${ }^{ \pm} \mathcal{D}^{\alpha} \tilde{u}^{\varepsilon}=\eta_{\varepsilon}{ }^{ \pm} \mathcal{D}^{\alpha} u \rightarrow{ }^{ \pm} \mathcal{D}^{\alpha} u$ in $L_{l o c}^{1}(\Omega)$ as $\varepsilon \rightarrow 0$. Hence, $\left\{\tilde{u}^{\varepsilon}\right\}$ is a desired sequence.

Step 2: Suppose that $\left\{u_{j}\right\}_{j=1}^{\infty} \subset C^{\infty}(\Omega)$ and $u_{j} \rightarrow u$ in $L^{1}(\Omega)$ and ${ }^{ \pm} \mathcal{D}^{\alpha} u_{j} \rightarrow v$ in $L_{l o c}^{1}(\Omega)$. Then for any $\varphi \in C_{0}^{\infty}(\Omega)$

$$
\left|\int_{\Omega}\left(u-u_{j}\right)(x)^{\mp} D^{\alpha} \varphi(x) d x\right| \leq M\left\|u-u_{j}\right\|_{L^{1}(\Omega)} \rightarrow 0 \quad \text { as } j \rightarrow \infty,
$$




$$
\begin{aligned}
\left|\int_{\Omega}\left({ }^{ \pm} \mathcal{D}^{\alpha} u_{j}-v\right)(x) \varphi(x) d x\right| & =\left|\int_{K}\left({ }^{ \pm} \mathcal{D}^{\alpha} u_{j}-v\right)(x) \varphi(x) d x\right| \\
& \leq M\left\|{ }^{ \pm} \mathcal{D}^{\alpha} u_{j}-v\right\|_{L^{1}(K)} \rightarrow 0 \text { as } j \rightarrow \infty
\end{aligned}
$$

because $K:=\operatorname{supp}(\varphi)$ is compact. It follows from the definition of weak fractional derivatives that

$$
\begin{aligned}
(-1)^{[\alpha]} \int_{\Omega} u(x)^{\mp} D^{\alpha} \varphi(x) d x & =(-1)^{[\alpha]} \lim _{j \rightarrow \infty} \int_{\Omega} u_{j}(x)^{\mp} D^{\alpha} \varphi(x) d x \\
& =\lim _{j \rightarrow \infty} \int_{\Omega}{ }^{ \pm} \mathcal{D}^{\alpha} u_{j}(x) \varphi(x) d x=\int_{\Omega} v(x) \varphi(x) d x .
\end{aligned}
$$

By the uniqueness of the weak fractional derivative, we conclude that $v={ }^{ \pm} \mathcal{D}^{\alpha} u$ almost everywhere. The proof is complete.

Corollary 3.7. Let $u \in L^{p}(\Omega)$ for $1 \leq p<\infty$. Then $v={ }^{ \pm} \mathcal{D}^{\alpha} u$ in $L_{\text {loc }}^{q}(\Omega)$ for $1 \leq q<\infty$ if and only if there exists a sequence $\left\{u_{j}\right\}_{j=1}^{\infty} \subset C^{\infty}(\Omega)$ such that $u_{j} \rightarrow u$ in $L^{p}(\Omega)$ and ${ }^{ \pm} \mathcal{D}^{\alpha} u_{j} \rightarrow v$ in $L_{l o c}^{q}(\Omega)$ as $j \rightarrow \infty$.

REMARK 3.3. The conclusion of the above corollary still holds if $L_{l o c}^{q}(\Omega)$ is replaced by $L^{q}(\Omega)$ in the statement.

3.3.2. The Infinite Domain Case. We now consider the case $\Omega=\mathbb{R}$. It turns out this case is significantly different from the finite interval case. In particular, it requires the construction of a compactly supported approximation sequence for each fractionally differentiable function, which turns out is quite complicated.

First, we establish the following analogue of Lemma 3.5. We refer the reader to [9, Lemma 3.3] for its proof.

Lemma 3.8. Suppose ${ }^{ \pm} \mathcal{D}^{\alpha} u \in L_{\text {loc }}^{1}(\mathbb{R})$ exists, then

$$
{ }^{ \pm} \mathcal{D}^{\alpha} u^{\varepsilon}=\eta_{\varepsilon} *{ }^{ \pm} \mathcal{D}^{\alpha} u \quad \text { a.e. in } \mathbb{R} \text {. }
$$

The next theorem gives a characterization of weak fractional derivatives on $\mathbb{R}$.

TheOrem 3.9. Suppose $u \in L^{1}(\mathbb{R})$. Then ${ }^{ \pm} \mathcal{D}^{\alpha} u=v \in L_{\text {loc }}^{1}(\mathbb{R})$ exists if and only if there exists a sequence $\left\{u_{j}\right\}_{j=1}^{\infty} \subset C_{0}^{\infty}(\mathbb{R})$ such that $u_{j} \rightarrow u$ in $L^{1}(\mathbb{R})$ and ${ }^{ \pm} \mathcal{D}^{\alpha} u_{j} \rightarrow v$ in $L_{\text {loc }}^{1}(\mathbb{R})$.

Proof. Step 1: Suppose that there exists $v \in L_{\text {loc }}^{1}(\mathbb{R})$ and $\left\{u_{j}\right\}_{j=1}^{\infty} \subset C_{0}^{\infty}(\mathbb{R})$ such that $u_{j} \rightarrow u$ in $L^{1}(\mathbb{R})$ and ${ }^{ \pm} \mathcal{D}^{\alpha} u_{j} \rightarrow v$ in $L_{\text {loc }}^{1}(\mathbb{R})$. We want to show $v={ }^{ \pm} \mathcal{D}^{\alpha} u$ almost everywhere. For any $\varphi \in C_{0}^{\infty}(\mathbb{R})$

$$
\begin{aligned}
\left|\int_{\mathbb{R}}\left(u-u_{j}\right)(x)^{\mp} D^{\alpha} \varphi(x) d x\right| & \leq\left.\int_{\mathbb{R}}\left|\left(u-u_{j}\right)(x)\right|\right|^{\mp} D^{\alpha} \varphi(x) \mid d x \\
& \leq\left\|u-u_{j}\right\|_{L^{1}(\mathbb{R})}\left\|^{\mp} D^{\alpha} \varphi\right\|_{L^{\infty}(\mathbb{R})} \rightarrow 0
\end{aligned}
$$

for $j \rightarrow \infty$ and for $K:=\operatorname{supp}(\varphi)$

$$
\begin{aligned}
\left|\int_{\mathbb{R}}\left(v-{ }^{ \pm} \mathcal{D}^{\alpha} u_{j}\right)(x) \varphi(x) d x\right| & \leq \int_{\mathbb{R}}\left|\left(v-{ }^{ \pm} \mathcal{D}^{\alpha} u_{j}\right)(x)\right||\varphi(x)| d x \\
& =\int_{K}\left|\left(v-{ }^{ \pm} \mathcal{D}^{\alpha} u_{j}\right)(x)\right||\varphi(x)| d x \\
& \leq\left\|v-{ }^{ \pm} \mathcal{D}^{\alpha} u_{j}\right\|_{L^{1}(K)}\|\varphi\|_{L^{\infty}(\mathbb{R})} \rightarrow 0
\end{aligned}
$$


for $j \rightarrow \infty$. From these inequalities and the definition of weak derivatives we get

$$
\begin{aligned}
(-1)^{[\alpha]} \int_{\mathbb{R}} u(x)^{\mp} D^{\alpha} \varphi(x) d x & =\lim _{j \rightarrow \infty}(-1)^{[\alpha]} \int_{\mathbb{R}} u_{j}(x)^{\mp} D^{\alpha} \varphi(x) d x \\
& =\lim _{j \rightarrow \infty} \int_{\mathbb{R}}{ }^{ \pm} \mathcal{D}^{\alpha} u_{j}(x) \varphi(x) d x=\int_{\mathbb{R}} v(x) \varphi(x) d x .
\end{aligned}
$$

By the uniqueness of the weak derivative, we deduce $v={ }^{ \pm} \mathcal{D}^{\alpha} u$ almost everywhere.

Step 2: Suppose that $u \in L^{1}(\mathbb{R})$ and $v:={ }^{ \pm} \mathcal{D}^{\alpha} u \in L_{\text {loc }}^{1}(\mathbb{R})$. We want to show that there exists $\left\{u_{j}\right\}_{j=1}^{\infty} \subset C_{0}^{\infty}(\mathbb{R})$ such that $u_{j} \rightarrow u$ in $L^{1}(\mathbb{R})$ and ${ }^{ \pm} \mathcal{D}^{\alpha} u_{j} \rightarrow v$ in $L_{\text {loc }}^{1}(\mathbb{R})$. To the end, let $\psi \in C^{\infty}(\mathbb{R})$ satisfy $\psi(t)=1$ if $t \leq 0$ and $\psi(t)=0$ if $t \geq 1$. For $j=1,2,3, \ldots$ let $\psi_{j} \in C_{0}^{\infty}(\mathbb{R})$ be defined by $\psi_{j}(x):=\psi(|x|-j)$. Let $u_{j}:=\eta_{\frac{1}{j}} *\left(\psi_{j} u\right)$. Then $u_{j} \in C_{0}^{\infty}(\mathbb{R})$ and $u_{j} \rightarrow u$ in $L^{1}(\mathbb{R})$ as $j \rightarrow \infty$. We also claim that ${ }^{ \pm} \mathcal{D}^{\alpha} u_{j} \rightarrow v$ in $L_{l o c}^{1}(\mathbb{R})$ as $j \rightarrow \infty$ and prove this conclusion below in the subsequent corollary. $\square$

Corollary 3.10. Suppose $u \in L^{p}(\mathbb{R})$ for $1 \leq p<\infty$. Then $v:={ }^{ \pm} \mathcal{D}^{\alpha} u \in$ $L_{\text {loc }}^{q}(\mathbb{R})$ for $1 \leq q<\infty$ if and only if there exists $\left\{u_{j}\right\}_{j=1}^{\infty} \subset C_{0}^{\infty}(\mathbb{R})$ such that $u_{j} \rightarrow u$ in $L^{p}(\mathbb{R})$ and ${ }^{ \pm} \mathcal{D}^{\alpha} u_{j} \rightarrow v$ in $L_{\text {loc }}^{q}(\mathbb{R})$.

Proof. Step 1: Same as Step 1 of the proof of Theorem 3.9.

Step 2: Suppose that $u \in L^{p}(\mathbb{R})$ for $1 \leq p<\infty$ and $v:={ }^{ \pm} \mathcal{D}^{\alpha} u \in L_{\text {loc }}^{q}(\mathbb{R})$. Let $\left\{u_{j}\right\}_{j=1}^{\infty}$ be the same as in the proof of Theorem 3.9 and $\varepsilon>0$. We now want to show that ${ }^{ \pm} \mathcal{D}^{\alpha} u_{j} \rightarrow v$ in $L_{l o c}^{q}(\mathbb{R})$ as $j \rightarrow \infty$.

By the assumption, we have $v={ }^{ \pm} \mathcal{D}^{\alpha} u \in L_{l o c}^{q}(\mathbb{R})$. For any fixed compact subset $K \subset \mathbb{R}$, choose $a, b \in \mathbb{R}$ such that $K \subset(a, b)$ finite. By the construction of $u_{j}$, we have ${ }^{ \pm} \mathcal{D}^{\alpha} u_{j}=\eta_{\frac{1}{j}}{ }^{ \pm} \mathcal{D}^{\alpha}\left(\psi_{j} u\right)$. Let $K_{j}:=\operatorname{supp}\left(\psi_{j}\right)$ and for every $\varphi \in C_{0}^{\infty}(\mathbb{R})$ with $\operatorname{supp}(\varphi) \subset K_{j}$ we have

$$
\begin{aligned}
\int_{K_{j}}{ }^{ \pm} \mathcal{D}^{\alpha}\left(\psi_{j} u\right)(x) \varphi(x) d x & =\int_{\mathbb{R}}{ }^{ \pm} \mathcal{D}^{\alpha}\left(\psi_{j} u\right)(x) \varphi(x) d x \\
& =\int_{\mathbb{R}}\left(\psi_{j} u\right)(x)^{\mp} D^{\alpha} \varphi(x) d x=\int_{K_{j}}\left(\psi_{j} u\right)(x)^{\mp} D^{\alpha} \varphi(x) d x .
\end{aligned}
$$

Hence, ${ }^{ \pm} \mathcal{D}^{\alpha}\left(\psi_{j} u\right)$ can be regarded as the weak fractional derivative of $\psi_{j} u$ over the domain $K_{j}$. It is due to this fact that we could use the product rule with remainder for fractional weak derivatives (to be proved in Theorem 3.13) to get

$$
{ }^{ \pm} \mathcal{D}^{\alpha}\left(\psi_{j} u\right)(x)=\psi_{j}(x)^{ \pm} \mathcal{D}^{\alpha} u(x)+\sum_{k=1}^{m} C_{k}{ }^{ \pm} I^{k-\alpha} u(x) D^{k} \psi_{j}(x)+{ }^{ \pm} R_{m}^{\alpha}\left(u, \psi_{j}\right)(x) .
$$

Therefore,

$$
\begin{gathered}
\left\|{ }^{ \pm} \mathcal{D}^{\alpha} u-{ }^{ \pm} \mathcal{D}^{\alpha} u_{j}\right\|_{L^{q}(K)}=\|{ }^{ \pm} \mathcal{D}^{\alpha} u-\eta_{\frac{1}{j}} *{ }^{ \pm} \mathcal{D}^{\alpha}\left(\psi_{j}, u\right) \\
=\|{ }^{ \pm} \mathcal{D}^{\alpha} u-\eta_{\frac{1}{j}} *\left(\psi_{j}{ }^{ \pm} \mathcal{D}^{\alpha} u+\sum_{k=1}^{m} C_{k}{ }^{ \pm} I^{\alpha} u D^{k} \psi_{j}+{ }^{ \pm} R_{m}^{\alpha}\left(u, \psi_{j}\right)\right) \\
\leq\|\|_{L^{q}(K)} \\
+\left\|\mathcal{D}^{\alpha} u-\eta_{\frac{1}{j}} * \psi_{j}{ }^{ \pm} \mathcal{D}^{\alpha} u\right\|_{L^{q}(K)}+\left\|\eta_{\frac{1}{j}} * \sum_{k=1}^{m} C_{k}{ }^{ \pm} I^{\alpha} u D^{k} \psi_{j}\right\|_{L^{q}(K)} \\
+\left\|{ }^{ \pm} R_{m}^{\alpha}\left(u, \psi_{j}\right)\right\|_{L^{q}(K)}
\end{gathered}
$$




$$
\begin{gathered}
\leq\left\|{ }^{ \pm} \mathcal{D}^{\alpha} u-\eta_{\frac{1}{j}} * \psi_{j}{ }^{ \pm} \mathcal{D}^{\alpha} u\right\|_{L^{q}(K)}+\sum_{k=1}^{m}\left\|\eta_{\frac{1}{j}} * C_{k}{ }^{ \pm} I^{\alpha} u D^{k} \psi_{j}\right\|_{L^{q}(K)} \\
+\left\|^{ \pm} R_{m}^{\alpha}\left(u, \psi_{j}\right)\right\|_{L^{q}(K)} .
\end{gathered}
$$

Then it suffices to show that each of the above three terms vanishes as $j \rightarrow \infty$.

Since ${ }^{ \pm} \mathcal{D}^{\alpha} u \in L^{q}(K)$, by the same arguments used to show that $u_{j} \rightarrow u$ in $L^{p}(\mathbb{R})$, we have that $\eta_{\frac{1}{j}} * \psi_{j}{ }^{ \pm} \mathcal{D}^{\alpha} u \rightarrow{ }^{ \pm} \mathcal{D}^{\alpha} u$ in $L^{q}(K)$. Hence, there exists $J_{1} \in \mathbb{N}$ such that for every $j \geq J_{1}$, we have that

$$
\left\|{ }^{ \pm} \mathcal{D}^{\alpha} u-\eta_{\frac{1}{j}} * \psi_{j}{ }^{ \pm} \mathcal{D}^{\alpha} u\right\|_{L^{q}(K)}<\frac{\varepsilon}{2} .
$$

Next, by construction, for set $K$, there exists $J_{2}:=J_{2}(K) \in \mathbb{N}$ so that for every $j \geq J_{2}, D^{k} \psi_{j}(x)=0$ for every $x \in(a, b)$. Therefore, for every $j \geq J_{2}$,

$$
\left\|\eta_{\frac{1}{j}} * C_{k}{ }^{ \pm} I^{k-\alpha} u D^{k} \psi_{j}\right\|_{L^{q}(K)} \leq\left\|C_{k}^{ \pm} I^{k-\alpha} u D^{k} \psi_{j}\right\|_{L^{q}((a, b))}=0 .
$$

Here we have used the fact that for each $k$ and $j \geq J_{2},{ }^{ \pm} I^{k-\alpha} u$ is finite on $(a, b)$. This of course is true since $u \in L^{p}(\mathbb{R})$. In fact, we need only that ${ }^{ \pm} I^{k-\alpha} u$ is finite on $(a, b)$ for $j=J_{2}$ since for all $j \geq J_{2}, D^{k} \psi_{j} \equiv 0$ in $(a, b)$.

Finally, it can be shown that the remainder term vanishes as $j \rightarrow \infty$. However, since the argument is rather lengthy, we omit it to save space and refer the reader to 9, Corollary 4.2] for a complete argument.

REMARK 3.4. The conclusion of the above corollary still holds if $L_{\text {loc }}^{q}(\mathbb{R})$ is replaced by $L^{q}(\mathbb{R})$ in the statement.

3.4. Basic Properties of Weak Fractional Derivatives. Similar to the classical calculus theory, we expect weak derivatives to satisfy certain properties and rules of calculus. As in the classical fractional calculus, many of the rules in the weak fractional calculus theory differ from their integer counterparts, which is expected. Below we list a few elementary properties for weak fractional derivatives.

Proposition 3.11. Let $\alpha, \beta>0, \lambda, \mu \in \mathbb{R}$, and $u, v$ be weakly differentiable to the appropriate order. Then the following properties hold.

(i) Linearity: ${ }^{ \pm} \mathcal{D}^{\alpha}(\lambda u+\mu v)=\lambda^{ \pm} \mathcal{D}^{\alpha} u+\mu^{ \pm} \mathcal{D}^{\alpha} v$.

(ii) Inclusivity: Let $0<\alpha<\beta<1$, suppose that $u$ is $\beta$ order weakly differentiable. Then $u$ is $\alpha$ order weakly differentiable.

(iii) Semigroup: suppose $0<\alpha, \beta, \alpha+\beta<1$ and ${ }^{ \pm} \mathcal{D}^{\alpha} u,{ }^{ \pm} \mathcal{D}^{\beta} u,{ }^{ \pm} \mathcal{D}^{\alpha+\beta} u \in L^{1}(\Omega)$, then ${ }^{ \pm} \mathcal{D}^{\alpha \pm} \mathcal{D}^{\beta} u={ }^{ \pm} \mathcal{D}^{\alpha+\beta} u$. Moreover, if $\alpha>1$, then ${ }^{ \pm} \mathcal{D}^{\alpha} u={ }^{ \pm} \mathcal{D}^{[\alpha]+\sigma} u=$ $\mathcal{D}^{[\alpha]}\left({ }^{ \pm} \mathcal{D}^{\sigma} u\right)$ with $\sigma:=\alpha-[\alpha]$.

(iv) Consistency: if $u$ is first-order weakly differentiable, then the $\alpha(<1)$ order weak derivative coincides with the first-order weak derivative in the limit as $\alpha \rightarrow 1$.

Proof. (i) It follows straightforwardly from a direction computation.

(ii) We shall postpone this proof until after the Fundamental Theorem of Weak Fractional Calculus (cf. Theorem 3.10) is established.

(iii) If $0<\alpha, \beta, \alpha+\beta<1$, by the definition we have

$$
\begin{aligned}
& \int_{\Omega}{ }^{ \pm} \mathcal{D}^{\alpha+\beta} u \varphi d x=\int_{\Omega} u^{\mp} D^{\alpha+\beta} \varphi d x \quad \forall \varphi \in C_{0}^{\infty}(\Omega), \\
& \int_{\Omega}{ }^{ \pm} \mathcal{D}^{\beta \pm} \mathcal{D}^{\alpha} u \varphi d x=\int_{\Omega}{ }^{ \pm} \mathcal{D}^{\alpha} u^{\mp} D^{\beta} \varphi d x \quad \forall \varphi \in C_{0}^{\infty}(\Omega) .
\end{aligned}
$$


Let $\left\{u_{j}\right\}_{j=1}^{\infty} \subset C^{\infty}(\Omega)$ such that $u_{j} \rightarrow u$ in $L^{1}(\Omega)$ and ${ }^{ \pm} D^{\alpha} u_{j} \rightarrow{ }^{ \pm} D^{\alpha} u$ in $L^{1}(\Omega)$, then using the integration by parts formula for Riemann-Liouville fractional order derivatives, we obtain

$$
\begin{aligned}
\int_{\Omega}{ }^{ \pm} \mathcal{D}^{\alpha} u^{\mp} D^{\beta} \varphi d x & =\lim _{j \rightarrow \infty} \int_{\Omega}{ }^{ \pm} D^{\alpha} u_{j}{ }^{\mp} D^{\beta} \varphi d x \\
& =\lim _{j \rightarrow \infty} \int_{\Omega} u_{j}{ }^{\mp} D^{\alpha \mp} D^{\beta} \varphi d x \\
& =\lim _{j \rightarrow \infty} \int_{\Omega} u_{j}{ }^{\mp} D^{\alpha+\beta} \varphi d x=\int_{\Omega} u^{\mp} D^{\alpha+\beta} \varphi d x .
\end{aligned}
$$

Combining (3.3)-(3.5) we get

$$
\int_{\Omega}{ }^{ \pm} \mathcal{D}^{\alpha+\beta} u \varphi d x=\int_{\Omega}{ }^{ \pm} \mathcal{D}^{\beta \pm} \mathcal{D}^{\alpha} u \varphi d x \quad \forall \varphi \in C_{0}^{\infty}(\Omega) .
$$

Thus, ${ }^{ \pm} \mathcal{D}^{\alpha+\beta} u={ }^{ \pm} \mathcal{D}^{\beta \pm} \mathcal{D}^{\alpha} u$ almost everywhere in $\Omega$.

If $\alpha>1$, set $m=[\alpha]$ and $\sigma=\alpha-m$. By the definition we get for any $\varphi \in C_{0}^{\infty}(\Omega)$,

$$
\begin{aligned}
\int_{\Omega} \mathcal{D}^{[\alpha]}\left({ }^{ \pm} \mathcal{D}^{\sigma} u\right) \varphi d x & =(-1)^{[\alpha]} \int_{\Omega}{ }^{ \pm} \mathcal{D}^{\sigma} u \mathcal{D}^{[\alpha]} \varphi d x=(-1)^{[\alpha]} \int_{\Omega} u^{\mp} \mathcal{D}^{\sigma} \mathcal{D}^{[\alpha]} \varphi d x \\
& =(-1)^{[\alpha]} \int_{\Omega} u^{\mp} \mathcal{D}^{\sigma+[\alpha]} \varphi d x=(-1)^{[\alpha]} \int_{\Omega} u^{\mp} \mathcal{D}^{\alpha} \varphi d x .
\end{aligned}
$$

Thus, ${ }^{ \pm} \mathcal{D}^{\alpha} u=\mathcal{D}^{[\alpha]}\left({ }^{ \pm} \mathcal{D}^{\sigma} u\right)$ almost everywhere in $\Omega$ and the assertion (iii) is proved.

(iv) It follows by the consistency of the classical fractional derivatives that for every $\varphi \in C^{\infty}(\Omega)$,

$$
\lim _{\alpha \rightarrow 1} \int_{\Omega}{ }^{ \pm} \mathcal{D}^{\alpha} u \varphi d x:=\lim _{\alpha \rightarrow 1}(-1)^{[\alpha]} \int_{\Omega} u^{\mp} D^{\alpha} \tilde{\varphi} d x=-\int_{\Omega} u D \tilde{\varphi} d x=: \int_{\Omega} \mathcal{D} u \varphi d x
$$

REMARK 3.5. We note that for $\alpha>1$, generally, ${ }^{ \pm} \mathcal{D}^{\alpha} u \neq{ }^{ \pm} \mathcal{D}^{\sigma} \mathcal{D}^{[\alpha]} u$, consequently, ${ }^{ \pm} \mathcal{D}^{\sigma} \mathcal{D}^{[\alpha]} u \neq \mathcal{D}^{[\alpha] \pm} \mathcal{D}^{\sigma} u$, in general.

We conclude this section by stating a general integration by parts formula in the case $\Omega=\mathbb{R}$.

Proposition 3.12. Let $\alpha>0,1 \leq p_{k} \leq \infty$ and $q_{k}=\frac{p_{k}}{p_{k}-1}$ for $k=1,2$. Suppose that $u \in L^{p_{1}}(\mathbb{R}), v \in L^{p_{2}}(\mathbb{R}),{ }^{ \pm} \mathcal{D}^{\alpha} u \in L^{q_{2}}(\mathbb{R})$, and ${ }^{\mp} \mathcal{D}^{\alpha} v \in L^{q_{1}}(\mathbb{R})$. Then there holds

$$
\int_{\mathbb{R}}{ }^{ \pm} \mathcal{D}^{\alpha} u v d x=(-1)^{[\alpha]} \int_{\mathbb{R}} u^{\mp} \mathcal{D}^{\alpha} v d x .
$$

Proof. By Corollary 3.10 we know that there exists a sequence $\left\{v_{j}\right\}_{j=1}^{\infty} \subset C_{0}^{\infty}(\mathbb{R})$ such that $v_{j} \rightarrow v$ in $L^{p_{2}}(\mathbb{R})$ and ${ }^{\mp} \mathcal{D}^{\alpha} v_{j} \rightarrow{ }^{\mp} \mathcal{D}^{\alpha} v$ in $L^{q_{1}}(\mathbb{R})$ as $j \rightarrow \infty$. By the definition of ${ }^{ \pm} \mathcal{D}^{\alpha} u$ we have

$$
\int_{\mathbb{R}}{ }^{ \pm} \mathcal{D}^{\alpha} u v_{j} d x=(-1)^{[\alpha]} \int_{\mathbb{R}} u^{\mp} \mathcal{D}^{\alpha} v_{j} d x .
$$

Setting $j \rightarrow \infty$ immediately infers (3.6). The proof is complete.

We note that in order to extend the above integration by parts formula to the finite domain case, it requires the notion of function traces, both function traces and the extended formula will be presented in 10 for functions in fractional Sobolev spaces. 
3.5. Product and Chain Rules for Weak Fractional Derivatives. In this subsection we present some product and chain rules for weak fractional derivatives, which are similar to those for classical fractional derivatives given in [9, Section 2.5].

TheOREM 3.13. Let $(a, b) \subset \mathbb{R}$ and $0<\alpha<1$. Suppose that $\psi \in C^{m+1}([a, b])$ for $m \geq 1$ and ${ }^{ \pm} \mathcal{D}^{\alpha} u \in L_{\text {loc }}^{1}((a, b))$ exists. Then ${ }^{ \pm} \mathcal{D}^{\alpha}(u \psi)$ exists and is given by

$$
\begin{gathered}
{ }^{ \pm} \mathcal{D}^{\alpha}(u \psi)(x)={ }^{ \pm} \mathcal{D}^{\alpha} u(x) \cdot \psi(x) \\
+\sum_{k=1}^{m} \frac{\Gamma(1+\alpha)}{\Gamma(1+k) \Gamma(1-k+\alpha)}{ }^{ \pm} I^{k-\alpha} u(x) D^{k} \psi(x) \\
+{ }^{ \pm} R_{m}^{\alpha}(u, \psi)(x) \quad \text { a.e. in }(a, b),
\end{gathered}
$$

where

$$
\begin{aligned}
& { }^{+} R_{m}^{\alpha}(u, \psi)(x)=\frac{(-1)^{m+1}}{m ! \Gamma(-\alpha)} \int_{x}^{b} \frac{u(y)}{(y-x)^{1+\alpha}} d y \int_{x}^{y} \psi^{(m+1)}(z)(z-x)^{m} d z \\
& { }^{-} R_{m}^{\alpha}(u, \psi)(x)=\frac{(-1)^{m+1}}{m ! \Gamma(-\alpha)} \int_{a}^{x} \frac{u(y)}{(x-y)^{1+\alpha}} d y \int_{y}^{x} \psi^{(m+1)}(z)(x-z)^{m} d z
\end{aligned}
$$

Proof. Let $\left\{u_{j}\right\}_{j=1}^{\infty} \subset C^{\infty}((a, b))$ so that $u_{j} \rightarrow u$ in $L^{1}((a, b))$ and ${ }^{ \pm} \mathcal{D}^{\alpha} u_{j} \rightarrow$ ${ }^{ \pm} \mathcal{D}^{\alpha} u$ in $L_{\text {loc }}^{1}((a, b))$. Consider the product $u_{j} \psi$, which belongs to $C((a, b))$, and $\varphi \in$ $C_{0}^{\infty}((a, b))$ with $\operatorname{supp}(\varphi):=(c, d) \subset(a, b)$. Since $u_{j} \rightarrow u$ in $\left.L^{1}(a, b)\right),{ }^{ \pm} I^{\sigma} u_{j} \rightarrow{ }^{ \pm} I^{\sigma} u$ in $L^{1}((a, b))$. Using this fact and [9, Theorem 2.3], we obtain

$$
\begin{aligned}
\int_{\Omega} u \psi^{\mp} D^{\alpha} \varphi d x & =\lim _{j \rightarrow \infty} \int_{\Omega} u_{j} \psi^{\mp} D^{\alpha} \varphi d x \\
& =\lim _{j \rightarrow \infty} \int_{\Omega}{ }^{ \pm} D^{\alpha}\left(u_{j} \psi\right) \cdot \varphi d x=\lim _{j \rightarrow \infty} \int_{\Omega^{\prime}}{ }^{ \pm} D^{\alpha}\left(u_{j} \psi\right) \cdot \varphi d x \\
& =\lim _{j \rightarrow \infty} \int_{\Omega^{\prime}}\left({ }^{ \pm} D^{\alpha} u_{j} \cdot \psi+\sum_{k=1}^{m} C_{k}{ }^{ \pm} I^{k-\alpha} u_{j} D^{k} \psi+{ }^{ \pm} R_{m}^{\alpha}\left(u_{j}, \psi\right)\right) \varphi d x \\
& =\int_{\Omega}\left({ }^{ \pm} \mathcal{D}^{\alpha} u \cdot \psi+\sum_{k=1}^{m} C_{k}{ }^{ \pm} I^{k-\alpha} u D^{k} \psi+{ }^{ \pm} R_{m}^{\alpha}(u, \psi)\right) \varphi d x,
\end{aligned}
$$

which implies that

$$
{ }^{ \pm} \mathcal{D}^{\alpha}(u \psi)={ }^{ \pm} \mathcal{D}^{\alpha} u \cdot \psi+\sum_{k=1}^{m} \frac{\Gamma(1+\alpha)}{\Gamma(k+1) \Gamma(1-k+\alpha)}{ }^{ \pm} I^{k-\alpha} u D^{k} \psi+{ }^{ \pm} R_{m}^{\alpha}(u, \psi)
$$

almost everywhere in $(a, b)$ with

$$
\begin{aligned}
& { }^{+} R_{m}^{\alpha}(u, \psi)(x)=\frac{(-1)^{m}}{m ! \Gamma(-\alpha)} \int_{x}^{b} \frac{u(y)}{(y-x)^{1+\alpha}} d y \int_{x}^{y} \psi^{(m+1)}(z)(z-x)^{m} d z \\
& { }^{-} R_{m}^{\alpha}(u, \psi)(x)=\frac{(-1)^{m+1}}{m ! \Gamma(-\alpha)} \int_{a}^{x} \frac{u(y)}{(x-y)^{1+\alpha}} d y \int_{y}^{x} \psi^{(m+1)}(z)(x-z)^{m} d z .
\end{aligned}
$$

The proof is complete.

REMARK 3.6. We also can prove another version of the product rules that do not include remainder terms. That version of the product rules will instead be written as infinite sums and require both functions are analytic. Because we do not wish to make 
such an assumption in our applications of the weak fractional derivative product rule, we omit that version of the product rules.

Based on the above product rules with $m=0$, we can easily obtain this following chain rules for weak fractional derivatives. We omit the proof because it is similar to the proof of [9, Theorem 2.4].

THEOREM 3.14. Let $(a, b) \subset \mathbb{R}$. Suppose that $\varphi \in C^{1}(\mathbb{R})$ such that $\varphi(0)=0$ and $f \in C((a, b))$. Then there hold

$$
{ }^{ \pm} \mathcal{D}^{\alpha} \varphi(f)(x)=\frac{\varphi(f)(x)}{f(x)}{ }^{ \pm} \mathcal{D}^{\alpha} f(x)+{ }^{ \pm} R_{0}^{\alpha}\left(f, \frac{\varphi(f)}{f}\right)(x) \quad \text { a.e. in }(a, b),
$$

where ${ }^{ \pm} R_{0}^{\alpha}(f, g)$ are defined by

$$
\begin{aligned}
& { }^{-} R_{0}^{\alpha}(f, g)(x)=\frac{-1}{\Gamma(-\alpha)} \int_{a}^{x} \frac{f(y)[g(x)-g(y)]}{(x-y)^{1+\alpha}} d y, \\
& { }^{+} R_{0}^{\alpha}(f, g)(x)=\frac{-1}{\Gamma(-\alpha)} \int_{x}^{b} \frac{f(y)[g(x)-g(y)]}{(y-x)^{1+\alpha}} d y .
\end{aligned}
$$

3.6. Fundamental Theorem of Weak Fractional Calculus (FTwFC). In this subsection, we aim to extend the FTcFC (see Theorem 2.13) to weakly fractionally differentiable functions. Similar to the FTcFC for classical fractional (RiemannLiouville) derivatives, the finite and infinite domain cases are significantly different, hence must be treated separately.

3.6.1. The Finite Interval Case. To establish the FTwFC on a finite domain, we first need to show the following crucial lift lemma.

Lemma 3.15. Let $\Omega \subset \mathbb{R}$ and $0<\alpha<1$. Suppose that $u \in L^{p}(\Omega)$ and ${ }^{ \pm} \mathcal{D}^{\alpha} u \in$ $L^{p}(\Omega)$ for some $1 \leq p<\infty$. Then ${ }^{ \pm} I^{1-\alpha} u \in W^{1,1}(\Omega)$.

Proof. Choose $\left\{u_{j}\right\}_{j=1}^{\infty} \subset C^{\infty}(\Omega)$ so that $u_{j} \rightarrow u$ in $L^{p}(\Omega)$ and ${ }^{ \pm} \mathcal{D}^{\alpha} u_{j} \rightarrow{ }^{ \pm} \mathcal{D}^{\alpha} u$ in $L^{p}(\Omega)$. Since ${ }^{ \pm} D^{\alpha} u_{j} \in L^{1}(\Omega)$, then ${ }^{ \pm} I^{1-\alpha} u_{j} \in W^{1,1}(\Omega)$. By the stability property of ${ }^{ \pm} I^{1-\alpha}$ we have

$$
\begin{aligned}
\left\|^{ \pm} I^{1-\alpha} u_{m}-{ }^{ \pm} I^{1-\alpha} u_{n}\right\|_{W^{1,1}(\Omega)}= & \left\|^{ \pm} I^{1-\alpha} u_{m}-{ }^{ \pm} I^{1-\alpha} u_{n}\right\|_{L^{1}(\Omega)} \\
& \quad+\left\|^{ \pm} D^{\alpha} u_{m}-{ }^{ \pm} D^{\alpha} u_{n}\right\|_{L^{1}(\Omega)} \\
\leq & C\left\|u_{m}-u_{n}\right\|_{L^{1}(\Omega)}+\left\|^{ \pm} \mathcal{D}^{\alpha} u_{m}-{ }^{ \pm} \mathcal{D}^{\alpha} u_{n}\right\|_{L^{1}(\Omega)} \\
& \rightarrow 0 \text { as } n, m \rightarrow \infty
\end{aligned}
$$

Hence, $\left\{{ }^{ \pm} I^{1-\alpha} u_{j}\right\}_{j=1}^{\infty}$ is a Cauchy sequence in $W^{1,1}(\Omega)$. Since $W^{1,1}(\Omega)$ is a Banach space, there exists $v \in W^{1,1}(\Omega)$ so that ${ }^{ \pm} I^{1-\alpha} u_{j} \rightarrow v$ in $W^{1,1}(\Omega)$.

It remains to show that $v={ }^{ \pm} I^{1-\alpha} u$. On noting that

$$
\begin{aligned}
\left\|v-{ }^{ \pm} I^{1-\alpha} u\right\|_{L^{1}(\Omega)} & \leq\left\|v-{ }^{ \pm} I^{1-\alpha} u_{j}\right\|_{L^{1}(\Omega)}+\left\|{ }^{ \pm} I^{1-\alpha} u_{j}-{ }^{ \pm} I^{1-\alpha} u\right\|_{L^{1}(\Omega)} \\
& \leq\left\|v-{ }^{ \pm} I^{1-\alpha} u_{j}\right\|_{L^{1}(\Omega)}+C\left\|u_{j}-u\right\|_{L^{1}(\Omega)} \rightarrow 0 \text { as } j \rightarrow \infty .
\end{aligned}
$$

Hence, $v={ }^{ \pm} I^{1-\alpha} u$ almost everywhere in $\Omega$. The proof is complete.

REMARK 3.7. Since $A C(\bar{\Omega})$ is isomorphic to $W^{1,1}(\Omega)$ in the $1 D$ case, the above lemma also implies that ${ }^{ \pm} I^{1-\alpha} u \in A C(\bar{\Omega})$. The above lemma shows that if $u \in$ ${ }^{ \pm} W^{\alpha, 1}(\Omega)$ (see the space definition in [10]), then the operator ${ }^{ \pm} I^{1-\alpha}$ lifts $u$ from ${ }^{ \pm} W^{\alpha, 1}(\Omega)$ into $W^{1,1}(\Omega)$. This result reinforces the characterization of weakly fractional differentiable functions as stated in Section 3.2. In particular, one can roughly 
think about weakly fractional differentiable functions as those whose classical fractional derivatives exist almost everywhere. This is (almost) exactly the same characterization for first order weakly differentiable functions (in 1D). Precisely, absolute continuity characterizes weakly differentiable functions and the absolute continuity of ${ }^{ \pm} I^{1-\alpha} u$ characterizes weakly fractional differentiable functions.

TheOREm 3.16. Let $\Omega \subset \mathbb{R}$ and $0<\alpha<1$. Suppose that $u \in L^{p}(\Omega)$ and ${ }^{ \pm} \mathcal{D}^{\alpha} u \in L^{p}(\Omega)$ for some $1 \leq p<\infty$. Then there holds

$$
u=c_{ \pm}^{1-\alpha} \kappa_{ \pm}^{\alpha}+{ }^{ \pm} I^{\alpha \pm} \mathcal{D}^{\alpha} u \quad \text { a.e. in } \Omega .
$$

Proof. Let $\left\{u_{j}\right\}_{j=1}^{\infty} \subset C^{\infty}(\Omega)$ so that $u_{j} \rightarrow u$ in $L^{p}(\Omega)$ and ${ }^{ \pm} \mathcal{D}^{\alpha} u_{j} \rightarrow{ }^{ \pm} \mathcal{D}^{\alpha} u$ in $L^{p}(\Omega)$; in particular, $u_{j}$ and its derivative converge in $L^{1}(\Omega)$. By Lemma 3.15. ${ }^{ \pm} I^{1-\alpha} u_{j} \rightarrow{ }^{ \pm} I^{1-\alpha} u$ in $W^{1,1}(\Omega) \cong A C(\bar{\Omega})$. Moreover, by the FTcFC we get

$$
u_{j}(x)=c_{j, \pm}^{1-\alpha} \kappa_{ \pm}^{\alpha}(x)+{ }^{ \pm} I^{\alpha \pm} D^{\alpha} u_{j}(x) .
$$

Thus,

$$
\begin{aligned}
& \left\|u-c_{ \pm}^{1-\alpha} \kappa_{ \pm}^{\alpha}-{ }^{ \pm} I^{\alpha \pm} D^{\alpha} u\right\|_{L^{1}(\Omega)} \\
& \quad \leq\left\|u-u_{j}\right\|_{L^{1}(\Omega)}+\left|c_{ \pm}^{1-\alpha}-c_{j, \pm}^{1-\alpha}\right|\left\|\kappa_{ \pm}^{\alpha}\right\|_{L^{1}(\Omega)}+\left\|^{ \pm} I^{\alpha}\left({ }^{ \pm} \mathcal{D}^{\alpha} u-{ }^{ \pm} D^{\alpha} u_{j}\right)\right\|_{L^{1}(\Omega)} \\
& \quad \leq\left\|u-u_{j}\right\|_{L^{1}(\Omega)}+\left|c_{ \pm}^{1-\alpha}-c_{j, \pm}^{1-\alpha}\right|\left\|\kappa_{ \pm}^{\alpha}\right\|_{L^{1}(\Omega)}+C\left\|^{ \pm} \mathcal{D}^{\alpha} u-{ }^{ \pm} D^{\alpha} u_{j}\right\|_{L^{1}(\Omega)} \\
& \quad \rightarrow 0 \quad \text { as } j \rightarrow \infty
\end{aligned}
$$

Therefore,

$$
u-c_{ \pm}^{1-\alpha} \kappa_{ \pm}^{\alpha}-{ }^{ \pm} I^{\alpha \pm} D^{\alpha} u=0 \quad \text { a.e. in } \Omega .
$$

The proof is complete.

REMARK 3.8. (a) We refer to Theorem 3.16 as the Fundamental Theorem of Weak Fractional Calculus (FTwFC) in this paper.

(b) The above FTwFC is an essential tool for studying weakly fractional differentiable functions, in particular, it will play a crucial role in proving compact and Sobolev embeddings and a fractional Poincaré inequality in [10].

To conclude this subsection, we would like to circle back to an unproven inclusion result for weak fractional derivatives which was alluded to in Proposition 3.11 part (ii). This then presents the first application of the FTwFC.

Proposition 3.17. Let $\Omega \subset \mathbb{R}$ and $0<\alpha<\beta<1$. Suppose that ${ }^{ \pm} \mathcal{D}^{\beta} u$ exists in $L^{1}(\Omega)$. Then ${ }^{ \pm} \mathcal{D}^{\alpha} u$ exists in $L^{1}(\Omega)$.

Proof. It follows by Theorem 3.16 that

$$
u=c_{ \pm}^{1-\beta} \kappa_{ \pm}^{\beta}+{ }^{ \pm} I^{\beta \pm} \mathcal{D}^{\beta} u \quad \text { a.e. in } \Omega \text {. }
$$

Then there holds

$$
\begin{aligned}
\int_{\Omega} u{ }^{\mp} D^{\alpha} \varphi d x & =\int_{\Omega}\left(c_{ \pm}^{1-\beta} \kappa_{ \pm}^{\beta}+{ }^{ \pm} I^{\beta \pm} \mathcal{D}^{\beta} u\right)^{\mp} D^{\alpha} \varphi d x \\
& =\int_{\Omega}{ }^{ \pm} D^{\alpha}\left(c_{ \pm}^{1-\beta} \kappa_{ \pm}^{\beta}+{ }^{ \pm} I^{\beta \pm} \mathcal{D}^{\beta} u\right) \varphi d x \\
& =\int_{\Omega}\left(c_{ \pm}^{1-\beta} \kappa_{ \pm}^{\beta-\alpha}+{ }^{ \pm} I^{\beta-\alpha \pm} \mathcal{D}^{\beta} u\right) \varphi d x
\end{aligned}
$$

Since a direct calculation shows that $v:=c_{ \pm}^{1-\beta} \kappa_{ \pm}^{\beta-\alpha}+{ }^{ \pm} I^{\beta-\alpha \pm} \mathcal{D}^{\beta} u \in L^{1}(\Omega)$, then the above identity implies that ${ }^{ \pm} \mathcal{D}^{\alpha} u$ exists and ${ }^{ \pm} \mathcal{D}^{\alpha} u=v$ almost everywhere in $\Omega$. The proof is complete. 
3.6.2. The Infinite Interval Case. Unlike the finite domain, the absence of any boundary in the infinite interval case $\Omega=\mathbb{R}$ allows for a cleaner statement of the FTwFC and a simpler proof.

TheOREm 3.18. Let $0<\alpha<1$. Suppose that $u, v \in L^{1}(\mathbb{R})$. If

$$
u={ }^{ \pm} I^{\alpha} v \text { a.e. in } \mathbb{R},
$$

then ${ }^{ \pm} \mathcal{D}^{\alpha} u=v$ almost everywhere. The converse is true under the additional assumption $u(x) \rightarrow 0$ almost everywhere as $|x| \rightarrow \infty$.

Proof. The assertion and the accompanying equation (3.11) follow from an application of the characterization theorem (cf. Theorem 3.9) for weak fractional derivatives and Theorem 2.17, $\mathrm{c}$

As was illustrated in the finite domain case and the infinite domain case for classical fractional derivatives, we can use the relation (3.11) to show a basic inclusion result for weak fractional derivatives.

Proposition 3.19. Let $0<\alpha<\beta<1$. Suppose that $u,{ }^{ \pm} \mathcal{D}^{\beta} u \in L^{1}(\mathbb{R})$. Then ${ }^{ \pm} \mathcal{D}^{\alpha} u$ exists in $L^{1}(\mathbb{R})$.

Proof. Apply the characterization theorem for weakly fractional differentiable functions on $\mathbb{R}$ (cf. Theorem 3.9) and the FTwFC (cf. Theorem 3.18), then pass limits.

4. Weak Fractional Derivatives of Distributions. The aim of this section is to introduce some weak fractional derivative notions for distributions. Like in the integer order case, such a notion is necessary in order to define fractional order weak derivatives for "all functions" including very rough ones and will also provide a useful tool for studying fractional order differential equations (cf. [11, 14, 23]).

The main difficulty for doing so is caused by the pollution effect of fractional order derivatives (and integrals), as a result, the standard test space $\mathscr{D}(\Omega):=C_{0}^{\infty}(\Omega)$ is not invariant under the mappings ${ }^{ \pm} D^{\alpha}$, instead, ${ }^{ \pm} D^{\alpha}(\mathscr{D}(\Omega)) \subset{ }^{ \pm} \mathscr{D}(\Omega):={ }^{\mp} C_{0}^{\infty}(\Omega)$ (see the definitions below). Hence, ${ }^{ \pm} D^{\alpha} \varphi$ become invalid test functions (or inputs) for a distribution $u \in \mathscr{D}^{\prime}(\Omega)$ although $\varphi \in \mathscr{D}(\Omega)$ is. To circumvent this difficulty, there are two approaches used in the literature. The first one, which is most popular [30, is to use different test spaces which are larger than the standard test space $\mathscr{D}(\Omega)$ so that the chosen test space is invariant under the mappings ${ }^{ \pm} D^{\alpha}$, and then to consider generalized functions (still called distributions) as continuous linear functionals on the chosen test space. The second approach is to extend the domain of a distribution $u \in \mathscr{D}^{\prime}(\Omega)$ without changing the standard test space $\mathscr{D}(\Omega)$ so that the extended distribution $\tilde{u}$ can take the inputs ${ }^{ \pm} D^{\alpha} \varphi$. In this section, we use both approaches although we give more effort to the second one because it covers general distributions in $\mathscr{D}^{\prime}(\Omega)$, not just a subclass of $\mathscr{D}^{\prime}(\Omega)$.

4.1. Test Spaces, Distributions and One-sided Distributions. We first recall some of the necessary function spaces and notions of convergence that are inherent to constructing a fractional derivative for distributions. We also introduce two new spaces of one-side compactly supported functions and establish some properties of the weak fractional derivative operators ${ }^{ \pm} \mathcal{D}^{\alpha}$ on the new spaces. Unless stated otherwise, in this section $\Omega$ denotes either a finite interval $(a, b)$ or the real line $\mathbb{R}$.

Definition 4.1. Let $\mathscr{D}(\Omega):=C_{0}^{\infty}(\Omega)$ which is equipped with the following topology (sequential convergence): given a sequence $\left\{\varphi_{k}\right\}_{k=1}^{\infty} \subset \mathscr{D}(\Omega)$ is said to converge to $\varphi \in \mathscr{D}(\Omega)$ if

(a) there exists a compact subset $K \subset \Omega$ such that $\operatorname{supp}\left(\varphi_{k}\right) \subset K$ for every $k$, 
(b) $D^{m} \varphi_{k} \rightarrow D^{m} \varphi$ uniformly in $K$ for each $m \geq 0$.

Let $\mathscr{D}^{\prime}(\Omega)$ denote the space of continuous linear functionals on $\mathscr{D}(\Omega)$, namely the dual space. Every functional in $\mathscr{D}^{\prime}(\Omega)$ is called a distribution.

DEFINITION 4.2. Define the following two spaces of one-side compactly supported functions:

$$
\begin{aligned}
& -\mathscr{D}(\Omega):=\left\{\varphi \in C^{\infty}(\Omega): \exists x_{0} \in \Omega, \varphi(x) \equiv 0 \forall x \leq x_{0}\right\}, \\
& +\mathscr{D}(\Omega):=\left\{\varphi \in C^{\infty}(\Omega): \exists x_{0} \in \Omega, \varphi(x) \equiv 0 \forall x \geq x_{0}\right\},
\end{aligned}
$$

which are equipped with the following topology: given a sequence $\left\{\varphi_{k}\right\}_{k=1}^{\infty} \subset \pm \mathscr{D}(\Omega)$, it is said to converge to $\varphi \in \pm \mathscr{D}(\Omega)$ if

(a) there exists an $x_{0} \in \Omega$ such that $\varphi_{k}(x) \equiv 0$ for all $x \leq x_{0}$ (or $x \geq x_{0}$ in the case of the right space) for $k \geq 1$,

(b) $D^{m} \varphi_{k} \rightarrow D^{m} \varphi$ uniformly in $\Omega$ for every $m \geq 0$.

Let ${ }^{ \pm} \mathscr{D}^{\prime}(\Omega)$ denote respectively the spaces of continuous linear functionals on ${ }^{ \pm} \mathscr{D}(\Omega)$, namely the dual spaces of $\pm \mathscr{D}(\Omega)$. Every functional in ${ }^{ \pm} \mathscr{D}^{\prime}(\Omega)$ is called a one-sided distribution.

LEMMA 4.3. $\mathscr{D}(\Omega)$ and ${ }^{ \pm} \mathscr{D}(\Omega)$ are complete topological vector spaces and $\mathscr{D}(\Omega) \subset$ $\pm \mathscr{D}(\Omega)$.

Recall that it was proved in Section 2.2 that ${ }^{ \pm} D^{\alpha}(\mathscr{D}(\Omega)) \subset{ }^{ \pm} \mathscr{D}(\Omega)$, Below we show that this inclusion is continuous.

Proposition 4.4. ${ }^{ \pm} \mathcal{D}^{\alpha}: \mathscr{D}(\Omega) \rightarrow{ }^{ \pm} \mathscr{D}(\Omega)$ are continuous.

Proof. We only give a proof for the left derivative ${ }^{-} \mathcal{D}^{\alpha}={ }^{-} D^{\alpha}$ because the other case follows similarly.

Let $\varphi_{k} \rightarrow \varphi$ in $\mathscr{D}(\Omega)$, we want to show that ${ }^{-} D^{\alpha} \varphi_{k} \rightarrow{ }^{-} D^{\alpha} \varphi$ in ${ }^{-} \mathscr{D}(\Omega)$. To the end, let $K \subset \subset \Omega$ be a compact subset so that $\operatorname{supp}\left(\varphi_{k}\right) \subset K$ for every $k \geq 0$ with $\varphi_{0} \equiv \varphi$, without loss of the generality, assume $K=\left[x_{0}, x_{1}\right] \subset \subset \Omega$. Then we have ${ }^{-} D^{\alpha} \varphi_{k} \equiv 0$ for every $x \leq x_{0}$ and $k \geq 0$, and for any integer $m \geq 0$ and $x>x_{0}$

$$
\begin{aligned}
\mid D^{m} & \left(-D^{\alpha} \varphi_{k}\right)(x)-D^{m}\left(-D^{\alpha} \varphi\right)(x) \mid \\
& =\left|\frac{d^{m}}{d x^{m}}\left[C_{\alpha} \frac{d}{d x} \int_{x_{0}}^{x} \frac{\varphi_{k}(y)}{(x-y)^{\alpha}} d y\right]-\frac{d^{m}}{d x^{m}}\left[C_{\alpha} \frac{d}{d x} \int_{x_{0}}^{x} \frac{\varphi(y)}{(x-y)^{\alpha}} d y\right]\right| \\
& =\left|C_{\alpha} \frac{d^{m+1}}{d x^{m+1}} \int_{x_{0}}^{x} \frac{\varphi_{k}(y)-\varphi(y)}{(x-y)^{\alpha}} d y\right| \\
& =\left|C_{\alpha} \int_{x_{0}}^{x} \frac{\varphi_{k}^{(m+1)}(y)-\varphi^{(m+1)}(y)}{(x-y)^{\alpha}} d y\right| \\
& \leq C_{\alpha} \int_{x_{0}}^{x_{1}} \frac{\left|\varphi_{k}^{(m+1)}(y)-\varphi^{(m+1)}(y)\right|}{|x-y|^{\alpha}} d y \\
& \leq \frac{|K|^{1-\alpha} C_{\alpha} \sup _{x \in K}\left|\varphi_{k}^{(m+1)}(x)-\varphi^{(m+1)}(x)\right| .}{1-\alpha}
\end{aligned}
$$

It follows by the uniform convergence of $\left\{\varphi_{k}\right\}_{k=1}^{\infty}$ that $D^{m-} D^{\alpha} \varphi_{k} \rightarrow D^{m-} D^{\alpha} \varphi$ uniformly in $\Omega$ for every $m$. The proof is complete. $\square$

The above proof also infers that the spaces ${ }^{ \pm} \mathscr{D}(\Omega)$ are invariant under the mapping ${ }^{ \pm} D^{\alpha}$, respectively.

Proposition 4.5. ${ }^{ \pm} \mathcal{D}^{\alpha}( \pm \mathscr{D}(\Omega)) \subset \pm \mathscr{D}(\Omega)$, respectively. Moreover, the inclusion is continuous.

REMARK 4.1. Without any added integrability condition (i.e. decay at $x=$ $\pm \infty)$, the inclusions of Proposition 4.5 may not be true when $\Omega=\mathbb{R}$. The smoothed 
(in an $\varepsilon$-neighborhood of $x=0$ ) Heaviside functions $H_{\varepsilon}(x)$ and $H_{\varepsilon}(-x)$ are two counterexamples. In fact, ${ }^{ \pm} D^{\alpha} \varphi$ may even not exist for some $\varphi \in{ }^{ \pm} \mathscr{D}(\mathbb{R})$.

Let $\mathcal{S}$ denote the space of Schwartz rapidly decaying functions defined in $\mathbb{R}$ (see 29] for the precise definition). Then we have

LEMma 4.6. The space $\mathcal{S}$ is invariant under the Fourier fractional order derivative operator, namely, ${ }^{\mathcal{F}} D^{\alpha}(\mathcal{S}) \subset \mathcal{S}$. Moreover, the inclusion is continuous.

Proof. Let $\varphi \in \mathcal{S}$, it is well known [1, 29] that $\hat{\varphi}:=\mathcal{F}[\varphi] \in \mathcal{S}$. Then $(i \xi)^{\alpha} \hat{\varphi} \in \mathcal{S}$, so is ${ }^{\mathcal{F}} D^{\alpha}(\varphi):=\mathcal{F}^{-1}\left[(i \xi)^{\alpha} \hat{\varphi}\right]$. The continuity of the inclusion can be proved in the same way as that in Proposition 4.4.

REMARK 4.2. It is easy to check that the Schwartz space $\mathcal{S}$ is not invariant under the mappings ${ }^{ \pm} \mathcal{D}^{\alpha}$, nor is it under ${ }^{ \pm} D^{\alpha}$. Consequently, the Fourier fractional derivatives and the Riemann-Liouville fractional derivatives may not coincide for functions in $\mathcal{S}$ in general. On the other hand, they do coincide for functions in $\mathscr{D}$ (see ([9], Proposition 2.2)). This fact is a main reason for and also validates the choice of test functions in the definition of weak fractional derivatives in Section 3.1.

4.2. Weak Fractional Derivatives for Compactly Supported Distributions. The goal of this subsection is to extend the notion of the weak fractional derivatives to distributions in $\mathscr{D}^{\prime}$ with compact supports. First, we recall the definition of supports for distributions.

DEFINITION 4.7. Let $u \in \mathscr{D}^{\prime}(\Omega), u$ is said to vanish on an open subset $O \subset \Omega$ if $u(\varphi)=0$ for all $\varphi \in C^{\infty}(\Omega)$ with $\operatorname{supp}(\varphi) \subset O$. Let $O_{\max }$ be a maximal open subset of $\Omega$ on which the distribution $u$ vanishes. The support of $u$ is defined as the complement of $O_{\max }$ in $\Omega$, that is, $\operatorname{supp}(u):=\Omega \backslash O_{\max }$. Moreover, $u$ is said to be compactly supported if $\operatorname{supp}(u)$ is a compact set.

The best known compactly supported distribution is the Dirac delta function $\delta_{0}$ which is defined by $\delta_{0}(\varphi)=\varphi(0)$ for any $\varphi \in \mathscr{D}(\mathbb{R})$. $\delta_{0}$ has the one point support $\{x=0\}$ and zero order.

Given a compact subset $K \subset \subset \Omega$, we also define the space

$$
\mathscr{D}_{K}^{\prime}(\Omega):=\left\{u \in \mathscr{D}^{\prime}(\Omega): \operatorname{supp}(u) \subseteq K\right\} .
$$

Lemma 4.8. Let $0<\alpha<1$ and $\psi, \varphi \in \mathscr{D}(\Omega)$. Then $\psi^{ \pm} D^{\alpha} \varphi \in \mathscr{D}(\Omega)$. Moreover, if $\varphi_{k} \rightarrow \varphi$ in $\mathscr{D}(\Omega)$, then $\psi^{ \pm} D^{\alpha} \varphi_{k} \rightarrow \psi^{ \pm} D^{\alpha} \varphi$ in $\mathscr{D}(\Omega)$.

Proof. Let $\psi, \varphi \in \mathscr{D}(\Omega)$. Recall that ${ }^{ \pm} D^{\alpha} \varphi \in C^{\infty}(\Omega)$. Then, $\psi^{ \pm} D^{\alpha} \varphi \in \mathscr{D}(\Omega)$. It remains to show the desired convergence result. Again, we only give a proof for the left space because the other case follows similarly.

Suppose that $\varphi_{k} \rightarrow \varphi$ in $\mathscr{D}(\Omega)$, then there exists a compact subset $K \subset \Omega$ so that $\operatorname{supp}\left(\varphi_{k}\right) \subset K$ for all $k \geq 1$. Without loss of the generality, assume $K=\left[x_{0}, x_{1}\right]$ and $K \cap \operatorname{supp}(\psi) \subset\left[x_{0}, x_{2}\right]$ for some $x_{2}>x_{0}$. Then, ${ }^{-} D^{\alpha} \varphi_{k} \equiv 0$ for $x \leq x_{0}$ and all $k \geq 1$ and $\psi \equiv 0$ for all $x>x_{2}$. Thus, for any integer $m \geq 1$ and $x_{0}<x<x_{2}$

$$
\begin{aligned}
\mid D^{m} & \left(\psi^{-} D^{\alpha} \varphi_{k}(x)\right)-D^{m}\left(\psi^{-} D^{\alpha} \varphi(x)\right) \mid \\
& =\left|\sum_{j=0}^{m}\left(\begin{array}{c}
m \\
j
\end{array}\right) \psi^{(m-j)} D^{j-} D^{\alpha} \varphi_{k}(x)-\sum_{j=0}^{m}\left(\begin{array}{c}
m \\
j
\end{array}\right) \psi^{(m-j)} D^{j-} D^{\alpha} \varphi(x)\right| \\
& \leq \sum_{j=0}^{m}\left(\begin{array}{c}
m \\
j
\end{array}\right)\left|\psi^{(m-j)}(x) D^{j-} D^{\alpha}\left(\varphi_{k}-\varphi\right)(x)\right| \\
& =\sum_{j=0}^{m}\left(\begin{array}{c}
m \\
j
\end{array}\right)\left|\psi^{(m-j)}(x)^{-} I^{1-\alpha}\left(\varphi_{k}^{(j+1)}-\varphi^{(j+1)}\right)(x)\right|
\end{aligned}
$$




$$
\leq \frac{C_{m} C_{\alpha}}{1-\alpha} \sup _{\substack{x_{0} \leq x \leq x_{2} \\ 1 \leq j \leq m}}\left(\left|\psi^{(m-j)}(x)\right| \cdot\left|\varphi_{k}^{(j+1)}(x)-\varphi^{(j+1)}(x)\right|\right) .
$$

By the uniform convergence of $\varphi_{k}$ to $\varphi$ in $\mathscr{D}(\Omega)$, we obtain the desired result.

The above lemma guarantees that $\psi^{ \pm} D^{\alpha} \varphi$ belongs to the standard test space $\mathscr{D}(\Omega)$ which removes most pollution contribution in ${ }^{ \pm} D^{\alpha} \varphi$ by using a compactly supported smooth (cutoff) function $\psi$.

For compactly supported distributions, there holds the following result, its proof can be found in [29, Theorem 6.24].

TheOREM 4.9. Let $u \in \mathscr{D}_{K}^{\prime}(\Omega)$. Then $u$ has a finite (integer) order $N(\geq 0)$ and can be uniquely extended to a continuous linear functional on $C^{\infty}(\Omega)$ which is given by

$$
\tilde{u}(\varphi):=u(\psi \varphi) \quad \forall \varphi \in C^{\infty}(\Omega),
$$

where $\psi \in C_{0}^{\infty}(\Omega)$ satisfying $\psi \equiv 1$ in $K$ is a partition of unity.

We note that the extension $\tilde{u}$ as a functional does not depend on the choice of the cut-off function $\psi$ (see [29] for a proof).

We now are ready to define weak fractional derivatives for compactly supported distributions in $\mathscr{D}^{\prime}(\Omega)$.

Definition 4.10. Let $\alpha>0$ and $u \in \mathscr{D}_{K}^{\prime}(\Omega)$. Define ${ }^{ \pm} \mathcal{D}^{\alpha} u: \mathscr{D}(\Omega) \rightarrow \mathbb{R}$ respectively by

$$
{ }^{ \pm} \mathcal{D}^{\alpha} u(\varphi):=(-1)^{[\alpha]} \tilde{u}\left({ }^{\mp} D^{\alpha} \varphi\right)=(-1)^{[\alpha]} u\left(\psi^{\mp} D^{\alpha} \varphi\right) \quad \forall \varphi \in \mathscr{D}(\Omega),
$$

where $\tilde{u}$ and $\psi$ are the same as in Theorem 4.9.

The next theorem shows that a compactly supported distribution $u \in \mathscr{D}_{K}^{\prime}(\Omega)$ has any order weak fractional derivative ${ }^{ \pm} \mathcal{D}^{\alpha} u$ which belongs to $\mathscr{D}^{\prime}(\Omega)$.

THEOREM 4.11. Let $\alpha>0$ and suppose $u \in \mathscr{D}_{K}^{\prime}(\Omega)$. Then

(i) ${ }^{ \pm} \mathcal{D}^{\alpha} u \in \mathscr{D}^{\prime}(\Omega)$. Moreover, if $K \subseteq[c, d] \subset \subset \Omega$, then $\operatorname{supp}\left({ }^{-} \mathcal{D}^{\alpha} u\right) \subseteq(-\infty, d] \cap$ $\Omega$ and $\operatorname{supp}\left({ }^{+} \mathcal{D}^{\alpha} u\right) \subseteq[c, \infty) \cap \Omega$.

(ii) Suppose that $\left\{u_{j}\right\}_{j=1}^{\infty} \subset \mathscr{D}_{K}^{\prime}(\Omega)$ such that $u_{j} \rightarrow u$ in $\mathscr{D}_{K}^{\prime}(\Omega)$, then ${ }^{ \pm} \mathcal{D}^{\alpha} u_{j} \rightarrow$ ${ }^{ \pm} \mathcal{D}^{\alpha} u$ in $\mathscr{D}^{\prime}(\Omega)$.

Proof. (i) The linearity of ${ }^{ \pm} \mathcal{D}^{\alpha} u$ is trivial. To show the continuity, it suffices to show that ${ }^{ \pm} \mathcal{D}^{\alpha} u$ is sequentially continuous at zero. To the end, let $\left\{\varphi_{k}\right\}_{k=1}^{\infty} \subset \mathscr{D}(\Omega)$ so that $\varphi_{k} \rightarrow 0$ in $\mathscr{D}(\Omega)$. It follows by Lemma 4.8 that

$$
{ }^{ \pm} \mathcal{D}^{\alpha} u\left(\varphi_{k}\right)=(-1)^{[\alpha]} u\left(\psi^{\mp} D^{\alpha} \varphi_{k}\right) \rightarrow u\left(\psi^{\mp} D^{\alpha}(0)\right)=0 \quad \text { as } k \rightarrow \infty .
$$

Since for any $\varphi \in \mathscr{D}(\Omega),{ }^{ \pm} \mathcal{D}^{\alpha} \varphi \in{ }^{ \pm} C_{0}^{\infty}(\Omega)$, then the supports of ${ }^{ \pm} \mathcal{D}^{\alpha} u$ pollute that of $u$ to the right/left accordingly.

(ii) Suppose that $u_{j} \rightarrow u$ in $\mathscr{D}^{\prime}(\Omega)$, we have for any $\varphi \in \mathscr{D}(\Omega)$

$$
{ }^{ \pm} \mathcal{D}^{\alpha} u_{j}(\varphi):=(-1)^{[\alpha]} u_{j}\left(\psi^{\mp} D^{\alpha} \varphi\right) \underset{j \rightarrow \infty}{\longrightarrow}(-1)^{[\alpha]} u\left(\psi^{\mp} D^{\alpha} \varphi\right)={ }^{ \pm} \mathcal{D}^{\alpha} u(\varphi) .
$$

Thus, ${ }^{ \pm} \mathcal{D}^{\alpha} u_{j} \rightarrow{ }^{ \pm} \mathcal{D}^{\alpha} u$ in $\mathscr{D}^{\prime}(\Omega)$ as $j \rightarrow \infty$. The proof is complete.

Proposition 4.12. Let $\alpha>0$ and suppose $u \in \mathscr{D}_{K}^{\prime}(\Omega)$. Then ${ }^{ \pm} \mathcal{D}^{\alpha} u \rightarrow \mathcal{D} u$ in $\mathscr{D}^{\prime}(\Omega)$ as $\alpha \rightarrow 1^{-}$and ${ }^{ \pm} \mathcal{D}^{\alpha} u \rightarrow \mathcal{D} u$ in $\mathscr{D}^{\prime}(\Omega)$ as $\alpha \rightarrow 1^{+}$.

Proof. For any $\varphi \in C_{0}^{\infty}(\Omega)$, we have

$$
{ }^{ \pm} \mathcal{D}^{\alpha} u(\varphi)=(-1)^{[\alpha]} u\left(\psi^{\mp} D^{\alpha} \varphi\right) \underset{\alpha \rightarrow 1^{-}}{\longrightarrow}-u(\psi D \varphi)=-u(D \varphi)=\mathcal{D} u(\varphi),
$$




$$
{ }^{ \pm} \mathcal{D}^{\alpha} u(\varphi)=(-1)^{[\alpha]} u\left(\psi^{\mp} D^{\alpha} \varphi\right) \underset{\alpha \rightarrow 1^{+}}{\longrightarrow}-u(\psi D \varphi)=-u(D \varphi)=\mathcal{D} u(\varphi) .
$$

Hence, the assertions hold. $\square$

Proposition 4.13. Let $\Omega=(a, b)$ and $0<\alpha<1$. Suppose that $u \in \mathscr{D}_{K}^{\prime}(\Omega)$ and $\eta \in C^{\infty}(\Omega)$, then there holds the following product rule:

$$
{ }^{ \pm} \mathcal{D}^{\alpha}(\eta u)=\eta^{ \pm} \mathcal{D}^{\alpha} u-\sum_{k=1}^{m} D^{k} \eta^{ \pm} I^{k-\alpha} u-C_{m, \alpha}\left(\eta^{(m+1)} * \mu_{ \pm}\right)\left(\kappa_{ \pm}^{-\alpha} * \psi u\right)
$$

where

$$
\begin{aligned}
C_{k, \alpha} & :=\frac{\Gamma(1+\alpha)}{\Gamma(k+1) \Gamma(1-k+\alpha)}, \\
{ }^{ \pm} I^{k-\alpha} u(\varphi) & :=C_{k, \alpha} u\left(\psi^{\mp} I^{k-\alpha} \varphi\right) \quad \forall \varphi \in \mathscr{D}(\Omega) .
\end{aligned}
$$

Proof. By the fractional order product rule, we have

$$
\begin{aligned}
{ }^{ \pm} \mathcal{D}^{\alpha}(\eta u)(\varphi): & =\eta u\left(\psi^{\mp} D^{\alpha} \varphi\right)=u\left(\eta \psi^{\mp} D^{\alpha} \varphi\right)=\left(u, \psi \eta^{\mp} D^{\alpha} \varphi\right) \\
& =u\left(\psi^{\mp} D^{\alpha}(\varphi \eta)\right)-u\left(\psi \sum_{k=1}^{m} C_{k, \alpha}{ }^{\mp} I^{k-\alpha} \varphi D^{k} \eta\right)-u\left(\psi^{\mp} R^{\alpha}(\varphi, \eta)\right) \\
& =: I-I I-I I I
\end{aligned}
$$

where

$$
{ }^{+} R_{m}^{\alpha}(\varphi, \eta):=\frac{(-1)^{m+1}}{m ! \Gamma(-\alpha)} \int_{x}^{b} \frac{\varphi(y)}{(y-x)^{1+\alpha}} d y \int_{x}^{y} \eta^{(m+1)}(z)(z-x)^{m} d z
$$

with a similar formula for ${ }^{-} R_{m}^{\alpha}(\varphi, \eta)$.

For terms $I$ and $I I$ we have

$$
\begin{aligned}
I: & =u\left(\psi^{\mp} D^{\alpha}(\varphi \eta)\right)={ }^{ \pm} \mathcal{D}^{\alpha} u(\eta \varphi)=\eta^{ \pm} \mathcal{D}^{\alpha} u(\varphi), \\
I I: & =u\left(\psi \sum_{k=1}^{m} C_{k, \alpha}{ }^{\mp} I^{k-\alpha} \varphi D^{k} \eta\right)=\sum_{k=1}^{m} C_{k, \alpha} u\left(\psi^{\mp} I^{k-\alpha} \varphi D^{k} \eta\right) \\
& =\sum_{k=1}^{m} C_{k, \alpha} D^{k} \eta u\left(\psi^{\mp} I^{k-\alpha} \varphi\right)=\sum_{k=1}^{m} D^{k} \eta^{ \pm} I^{k-\alpha} u(\varphi) .
\end{aligned}
$$

Finally, to simplify term $I I I$, we rewrite the remainder formula as follows:

$$
\begin{aligned}
{ }^{+} R_{m}^{\alpha}(\varphi, \eta) & :=\frac{(-1)^{m+1}}{m ! \Gamma(-\alpha)} \int_{x}^{b} \int_{x}^{y} \frac{\varphi(y)}{(y-x)^{1+\alpha}} \eta^{(m+1)}(z)(z-x)^{m} d z d y \\
& =\frac{(-1)^{m+1}}{m ! \Gamma(-\alpha)} \int_{x}^{b} \frac{\varphi(y)}{(y-x)^{1+\alpha}}\left(\eta^{(m+1)} * \mu_{+}\right)(y) d y \\
& =C_{m, \alpha}\left(\varphi\left(\eta^{(m+1)} * \mu_{+}\right) * \kappa_{+}^{-\alpha}\right)(x) .
\end{aligned}
$$

Then we have

$$
\begin{aligned}
I I I: & =u\left(\psi^{+} R^{\alpha}(\varphi, \eta)\right) \\
& =u\left(C_{m, \alpha} \psi\left(\varphi\left(\eta^{(m+1)} * \mu_{+}\right) * \kappa_{+}^{-\alpha}\right)\right)
\end{aligned}
$$




$$
\begin{aligned}
& =C_{m, \alpha} u\left(\psi\left(\varphi\left(\eta^{(m+1)} * \mu_{+}\right) * \kappa_{+}^{-\alpha}\right)\right) \\
& =C_{m, \alpha}\left(\kappa_{+}^{-\alpha} * \psi u\right)\left(\varphi\left(\eta^{(m+1)} * \mu_{+}\right)\right) \\
& =C_{m, \alpha}\left(\eta^{(m+1)} * \mu_{+}\right)\left(\kappa_{+}^{-\alpha} * \psi u\right)(\varphi) . \quad=C_{m, \alpha}\left(\eta^{(m+1)} * \mu_{+}\right) \cdot\left(\kappa_{+}^{-\alpha} *(\psi u)\right)(\varphi) .
\end{aligned}
$$

The desired formula (4.1) follows from combining the above three identities. The proof is complete.

4.3. Weak Fractional Derivatives for Distributions on Finite Intervals. In the previous subsection we introduce a fractional order derivative notion for compactly supported distributions in $\mathscr{D}_{K}^{\prime}(\Omega)$. The aim of this subsection is to introduce a fractional derivative notion for general distributions in $\mathscr{D}^{\prime}(\Omega)$ when $\Omega=(a, b)$ is finite. We shall address the case $\Omega=\mathbb{R}$ in the next subsection.

First, we consider the class of one sided generalized functions in ${ }^{ \pm} \mathscr{D}^{\prime}(\Omega):=$ $\left({ }^{ \pm} \mathscr{D}(\Omega)\right)^{\prime}$, which are proper subspaces of $\mathscr{D}^{\prime}(\Omega)$. By Proposition 4.5 we know that ${ }^{ \pm} \mathscr{D}(\Omega)$ are respectively invariant under the mappings ${ }^{ \pm} D^{\alpha}$. This fact then makes defining ${ }^{ \pm} \mathcal{D}^{\alpha} u$ for $u \in{ }^{ \pm} \mathscr{D}^{\prime}(\Omega)$ a trivial task.

Definition 4.14. Let $\alpha>0$ and $u \in{ }^{ \pm} \mathscr{D}^{\prime}(\Omega)$. Define ${ }^{ \pm} \mathcal{D}^{\alpha} u:{ }^{ \pm} \mathscr{D}(\Omega) \rightarrow \mathbb{R}$ respectively by

$$
{ }^{ \pm} \mathcal{D}^{\alpha} u(\varphi):=(-1)^{[\alpha]} u\left({ }^{\mp} D^{\alpha} \varphi\right) \quad \forall \varphi \in{ }^{ \pm} \mathscr{D}(\Omega) .
$$

Clearly, ${ }^{ \pm} \mathcal{D}^{\alpha} u$ is well defined and ${ }^{ \pm} \mathcal{D}^{\alpha} u \in{ }^{ \pm} \mathscr{D}^{\prime}(\Omega)$, respectively. It also can be shown that many other properties hold for the fractional order derivative operators ${ }^{ \pm} \mathcal{D}^{\alpha}$ on the one sided generalized function spaces ${ }^{ \pm} \mathscr{D}^{\prime}(\Omega)$. We leave the verification to the interested reader.

To define fractional order derivatives for distributions in $\mathscr{D}^{\prime}(\Omega) \backslash^{-} \mathscr{D}^{\prime}(\Omega) \cup^{+} \mathscr{D}^{\prime}(\Omega)$, we need to construct "good" extensions for any distribution $u \in \mathscr{D}^{\prime}(\Omega)$ to ${ }^{-} \mathscr{D}^{\prime}(\Omega)$ and $+\mathscr{D}^{\prime}(\Omega)$. This will be done below by using the partition of unity theorem to define $u(\varphi):=\sum_{j=1}^{\infty} u\left(\psi_{j} \varphi\right)$ for any $\varphi \in{ }^{ \pm} \mathscr{D}(\Omega)$.

Let $\left\{I_{\beta}\right\}$ be a family of open subintervals of $(a, b)$ which forms a covering of $\Omega$. By the partition of unity theorem (cf. [29]), there exists a subsequence $\left\{I_{j}\right\}_{j=1}^{\infty} \subset\left\{I_{\beta}\right\}$ and a partition of the unity $\left\{\psi_{j}\right\}_{j=1}^{\infty}$ subordinated to $\left\{I_{j}\right\}_{j=1}^{\infty}$, namely, $\psi_{j} \in C_{0}^{\infty}\left(I_{j}\right)$ for $j \geq 1$ and $\sum \psi_{j}(x) \equiv 1$ on every compact subset $K$ of $\Omega$ and the sum is a finite sum for every $x \in K$.

Definition 4.15. Let $\alpha>0$ and $u \in \mathscr{D}^{\prime}(\Omega)$. Define ${ }^{ \pm} \mathcal{D}^{\alpha} u: \mathscr{D}(\Omega) \rightarrow \mathbb{R}$ respectively by

$$
{ }^{ \pm} \mathcal{D}^{\alpha} u(\varphi):=(-1)^{[\alpha]} \sum_{j=1}^{\infty} u\left(\psi_{j}{ }^{\mp} D^{\alpha} \varphi\right) \quad \forall \varphi \in \mathscr{D}(\Omega) .
$$

We claim that ${ }^{ \pm} \mathcal{D}^{\alpha} u$ is well defined and ${ }^{ \pm} \mathcal{D}^{\alpha} u \in \mathscr{D}^{\prime}(\Omega)$, respectively. We leave the verification to the interested reader.

4.4. Weak Fractional Derivatives for Distributions on $\mathbb{R}$. To define RiemannLiouville fractional order derivatives for distributions in $\mathscr{D}^{\prime}(\mathbb{R})$ is more complicated than in ${ }^{ \pm} \mathscr{D}^{\prime}(\Omega)$; the complication is due to the fact that the kernel functions $\kappa_{ \pm}^{\alpha} \notin$ $L^{1}(\mathbb{R})$ and the pollutions of ${ }^{ \pm} \mathcal{D}^{\alpha} \varphi(x)$ for $\varphi \in \mathscr{D}(\mathbb{R})$ do not decay fast enough when $x \rightarrow \pm \infty$.

We first consider the simpler case of Fourier fractional order derivatives for tempered distributions in $\mathcal{S}^{\prime}(\mathbb{R})$. By Proposition 4.6 we know that the Schwartz space 
$\mathcal{S}(\mathbb{R})$ is invariant under the Fourier derivative operator ${ }^{\mathcal{F}} D^{\alpha}$. This allows us easily to define Fourier fractional derivatives for tempered distributions as follows.

Definition 4.16. Let $\alpha>0$ and $u \in \mathcal{S}^{\prime}(\mathbb{R})$. Define ${ }^{\mathcal{F}} \mathcal{D}^{\alpha} u: \mathcal{S}(\mathbb{R}) \rightarrow \mathbb{R}$ by

$$
{ }^{\mathcal{F}} \mathcal{D}^{\alpha} u(\varphi):=(-1)^{[\alpha]} u\left({ }^{\mathcal{F}} D^{\alpha} \varphi\right) \quad \forall \varphi \in \mathcal{S}(\mathbb{R}) .
$$

It is easy to verify that ${ }^{\mathcal{F}} \mathcal{D}^{\alpha} u$ is well defined and ${ }^{\mathcal{F}} \mathcal{D}^{\alpha} u \in \mathcal{S}^{\prime}(\mathbb{R})$. It also can be shown that many other properties hold for the fractional order derivative operator ${ }^{\mathcal{F}} \mathcal{D}^{\alpha}$ on the space of tempered distributions $\mathcal{S}^{\prime}(\mathbb{R})$. We leave the verification to the interested reader.

To define fractional order derivatives for distributions in $\mathscr{D}^{\prime}(\mathbb{R}) \backslash \mathcal{S}^{\prime}(\mathbb{R})$, we need to extend the domain of $u \in \mathscr{D}^{\prime}(\mathbb{R})$ from $\mathscr{D}(\mathbb{R})$ to $\mathcal{S}(\mathbb{R})$ (or ${ }^{ \pm} \mathscr{D}(\mathbb{R})$ ). Again, this will be done by using the partition of the unity theorem as seen above to define $u(\varphi):=\sum_{j=1}^{\infty} u\left(\psi_{j} \varphi\right)$ for any $\varphi \in{ }^{ \pm} \mathscr{D}(\mathbb{R})$.

Let $\left\{I_{\beta}\right\}$ be a family of open finite subintervals of $\mathbb{R}$ which forms a covering of $\mathbb{R}$. By the partition of unity theorem (cf. [29]), there exists a subsequence $\left\{I_{j}\right\}_{j=1}^{\infty} \subset\left\{I_{\beta}\right\}$ and a partition of the unity $\left\{\psi_{j}\right\}_{j=1}^{\infty}$ subordinated to $\left\{I_{j}\right\}_{j=1}^{\infty}$, namely, $\psi_{j} \in C_{0}^{\infty}\left(I_{j}\right)$ for $j \geq 1$ and $\sum \psi_{j}(x) \equiv 1$ on every compact subset $K$ of $\mathbb{R}$ and the sum is a finite sum for every $x \in K$.

Definition 4.17. Let $\alpha>0$ and $u \in \mathscr{D}^{\prime}(\mathbb{R})$. Define ${ }^{ \pm} \mathcal{D}^{\alpha} u: \mathscr{D}(\mathbb{R}) \rightarrow \mathbb{R}$ respectively by

$$
{ }^{ \pm} \mathcal{D}^{\alpha} u(\varphi):=(-1)^{[\alpha]} \sum_{j=1}^{\infty} u\left(\psi_{j}{ }^{\mp} D^{\alpha} \varphi\right) \quad \forall \varphi \in \mathscr{D}(\mathbb{R}) .
$$

We claim that ${ }^{ \pm} \mathcal{D}^{\alpha} u$ is well defined and ${ }^{ \pm} \mathcal{D}^{\alpha} u \in \mathscr{D}^{\prime}(\mathbb{R})$, respectively. Again, we leave the verification to the interested reader.

5. Conclusion. In this paper we first recalled various definitions of classical fractional derivatives and gave a new interpretation of the classical theory from a different perspective, and especially emphasized the importance of the Fundamental Theorem of Classical Fractional Calculus (FTcFC) and its ramifications in the classical theory. We then presented a self-contained new theory of weak fractional differential calculus. The crux of this new theory is the introduction of a weak fractional derivative notion which is a natural generalization of integer order weak derivatives; it also helps to unify multiple existing fractional derivative definitions and has the potential to be easily extended to higher dimensions. Various calculus rules including a Fundamental Theorem of Weak Fractional Calculus (FTwFC), product and chain rules, and integration by parts formulas were established for weak fractional derivatives and relationships with existing classical fractional derivatives were also obtained. This weak fractional differential calculus theory lays down the ground work for developing a new fractional order Sobolev space theory in a companion paper [10]. Furthermore, the notion of weak fractional derivatives was systematically extended to general distributions instead of only to some special distributions as done in the literature. It is expected (and our hope, too) that these newly developed theories of weak fractional differential calculus and fractional order Sobolev spaces will lay down a solid theoretical foundation for systematically and rigorously developing a fractional calculus of variations theory and a fractional PDE theory as well as their numerical solutions. Moreover, we hope this work will stimulate more research on and applications of fractional calculus and fractional differential equations in the near future. 


\section{REFERENCES}

[1] R.A. Adams, Sobolev Spaces, Pure and Applied Mathematics, Vol. 65. Academic Press, New York, 1975.

[2] M.A. Bassam, Some properties of Holmgren-Riesz transform, Ann. Scuola Norm. Super., Pisa, 1961.

[3] H. Brezis, Functional Analysis, Sobolev Spaces and Partial Differential Equations, Springer, New York, 2011.

[4] S. DAs, Functional Fractional Calculus, Springer, Berlin, 2011.

[5] C. Li and W. Deng, Remarks on fractional derivatives, Appl. Math. Comput., 187(2), 777-784, 2007.

[6] Q. Du, Nonlocal Modeling, Analysis, and Computation, SIAM, Philadelphia, 2019.

[7] V. ERvin AND J. P. RoOp, Variational formulation for the stationary fractional advection dispersion equation, Numer. Methods for PDEs, 22(2), 558-576, 2006.

[8] L. C. Evans, Partial Differential Equations, AMS, Providence, RI, 2010.

[9] X. Feng And M. Sutton, A new theory of fractional differential calculus and fractional Sobolev spaces: One-dimensional case, arXiv:2004.10833.

[10] X. Feng And M. Sutton, New fractional Sobolev spaces in one dimension, in preparation.

[11] X. Feng And M. Sutton, A new theory of fractional calculus of variations and fractional differential equations, in preparation.

[12] X. Feng And M. Sutton, Finite element methods for approximating weak fractional derivatives and fractional differential equations, in preparation.

[13] D. Gilbarg and N. Trudinger, Elliptic Partial Differential Equations of Second Order, Springer, New York, 1998.

[14] B. Guo, X. Pu, And F. Huang, Fractional Partial Differential Equations and Their Numerical Solutions, World Scientific Publishing Co., London, 2015.

[15] M. Herzallah, Notes on some fractional calculus operators and their properties, J. Fract. Calc. Appli, 5(3s), 1-10, 2014.

[16] R. Hilfer, Applications of Fractional Calculus in Physics, World Scientific Press, 2000.

[17] R. Khalil, M.Al Horani, A. Youse,and M. Sababheh, A new definition of fractional derivative, J. Computat. Applied Math, 264, 65-70, 2014.

[18] A. A. Kilbas, H. M. Srivastava, and J. J. Trujillo, Theory and Applications of Fractional Differential Equations, Elsevier, 2006.

[19] M. KLIMEK, On Solutions of Linear Fractional Differential Equations of Variational Type, The Publishing Office of Czestochowa University of Technology, 2009.

[20] C. Li, D. Qian, And Y. Chen, On Riemann-Liouville and Caputo derivatives, Discet. Dyn. in Nature and Society, 1-15, 2011.

[21] J. Liouville, Memoire sur le calcul des differentielles a indices quelconques, Journal de l'Ecole Royale Polytechnique, Extraits du Tome, 13. Sect. 21, 71 - 162, 1832.

[22] A. B. Malinowska, T. OdziJewicz, and D. F. M. Torres, Advanced Methods in the Fractional Calculus of Variations, Springer, Berlin, 2015.

[23] M. Meerschaert And A. Sikorskiz, Stochastic Models for Fractional Calculus, de Gruyter, 2012.

[24] J. Munkhammar, Riemann-Liouville Fractional derivatives and the Taylor-Riemann series, 2004

[25] N. G. Meyers and J. Serrin, $H=W$, Proceed. Nati. Acad. of Sci., 51(6), 1055-1056, 1964.

[26] E. Di Nezza, G. Palatucci, and E. Valdinoci, Hitchhiker's guide to the fractional Sobolev spaces, Bulletin des sciences mathematique, 136(5), 521-573, 2012.

[27] T. OsLer, Leibniz rule for fractional derivatives generalized and an application to infinite series, SIAM J. Appl. Math., 18(3), 658-674, 1970.

[28] I. Podlubny, Fractional Differential Equations, Mathematics in science and engineering, Vol. 198, Academic Press, New York, 1999.

[29] W. Rudin, Fractional Analysis, McGraw-Hill, New York, 1991.

[30] S. G. Samko, A. A. Kilbas, And O. I. Marichev, Fractional Integrals and Derivatives: Theory and Applications, GRC Press, 1993.

[31] P. R. Stinga And M. VAughan One-sided fractional derivatives, fractional Laplacians, and weighted Sobolev spaces, Nonl. Anal., 193, https://doi.org/10.1016/j.na.2019.04.004, 2020.

[32] V. Tarasov, No violation of the Leibniz rule. No fractional derivative, Comm. Nonl. Sci. Numer. Simul., 18, 2945-2948, 2013.

[33] V. Tarasov, On chain rule for fractional derivatives., Comm. Nonl. Sci. Numer. Simul., 30, $1-3,2016$ 